\title{
EXPERIMENTAL PARTICLE PHYSICS
}

\author{
Progress Report for Period \\ September 16, 1991 - September 30, 1992 \\ R.I. Steinberg and C.E. Lane \\ Drexel University \\ Department of Physics \\ Philadelphia, PA 19104
}

September 1992

\begin{abstract}
NOTICE
This report was prepared as an account of work sponsored by the United States Government. Neither the United States nor the Department of Energy, nor any of their employees, nor any of their contractors, subcontractors, or their employees, makes any warranty, express or implied, or assumes any legal liability or responsibility for the accuracy, completeness, or usefulness of any information, apparatus, product or process disclosed or represents that its use would not infringe privately-owned rights.
\end{abstract}

Prepared for:

THE U. S. DEPARTMENT OF ENERGY

Contract No. DE-FG02-91ER40615 


\section{Contents}

1 Introduction 2

2 The MACRO Experiment $\quad 7$

2.1 MACRO Liquid Scintillator .............. 7

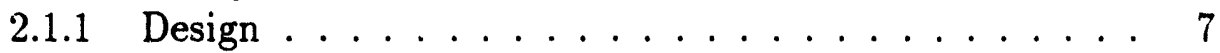

2.1.2 Production and Testing ............ 9

2.2 MACRO Fast Monopole Electronics . . . . . . . . . . . . . 10

2.2 .1 Introduction . . . . . . . . . . . . 10

2.2.2 MACRO Fast Monopole Physics . . . . . . . . . . 10

2.2 .3 Trigger Design ... . . . . . . . . . . 12

2.2 .4 Implementation . . . . . . . . . . . . . 17

2.3 MACRO Data Analysis . . . . . . . . . . . . . . 25

2.4 Shifts and On-Site Work . . . . . . . . . . . 26

3 Neutrino Oscillations $\quad 27$

3.1 Introduction . . . . . . . . . . . . . 27

3.2 Experimental Searches for Neutrino Mass . . . . . . . . 27

3.3 Neutrino Vacuum Flavor Oscillations . . . . . . . . . . . 27

3.4 Reactor Neutrino Oscillation Experiments . . . . . . . . . 28

4 The Chooz Neutrino Oscillation Experiment 31

4.1 Introduction . . . . . . . . . . . . . . . 31

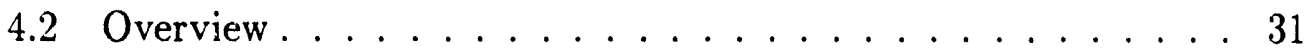

4.3 Event Rate and Anticipated Result . . . . . . . . . . . 34

5 The Perry Experiment $\quad 35$

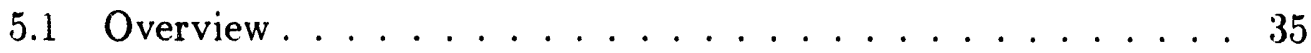

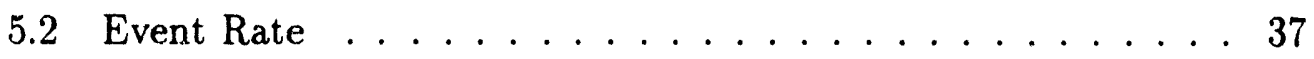

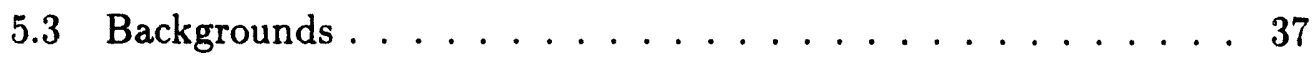

5.4 Event Generation and Reconstruction . . . . . . . . . . . . . 39

5.5 Background Tests . . . . . . . . . . . . . . 42

6 SSC-Related Research $\mathbf{4 3}$

6.1 Introduction . . . . . . . . . . . . . . . . . 43

6.1 .1 SSC R\&D . . . . . . . . . . . . . . 43

6.1.2 GEM Electronics ............... 43 
7 Recent Drexel Particle Physics Publications

References 


\section{List of Figures}

1 Schematic View of the MACRO Experiment . . . . . . 8

2 Astrophysical and Experimental Bounds on the Monopole Flux 11

3 Expected Scintillation Yield for a Magnetic Monopole. . . . . 11

4 Experimental Signature of a Monopole . . . . . . . . . . . 12

5 Comparison of Signatures in MACRO . . . . . . . . . . . 13

$6 \quad$ Fast Moriupole Trigger Logic . . . . . . . . . . . . . . . . 15

7 Coverage of the Triggers . . . . . . . . . . . . 16

8 Schematic for the Fast Linear 8:1 Fanin . . . . . . . . . . . 18

9 Response Function of Production Fanin . . . . . . . . . . . . 19

10 Response Function of 8 Inputs of Fanin . . . . . . . . . . . . 20

11 Block Diagram of FMT Module . . . . . . . . . . . . 22

12 Calibration and Testing Scheme for Fast Monopole Triggers . 24

13 Exclusion Plot . . . . . . . . . . . . . . . . . . . . 30

14 Muon Depth-Intensity Plot . . . . . . . . . . . . . . 33

15 The Perry Underground Neutrino Oscillation Experiment . . 36

16 Distance between Reconstructed and Actual Positions . . . . . 40

17 Reconstructed Neutron Capture Energy . . . . . . . . . . . . 41

\section{List of Tables}

1 Parameters of the Chooz Scintillation Detector . . . . . . . . 32

2 Inverse Beta Decay Event Rate in the Chooz Experiment . . . 34

3 Inverse Beta Decay Event, Rate in the Perry Experiment . . . 37

4 Input Parameters for Event Reconstruction Program . . . . . 39 


\begin{abstract}
The goals of this research are the experimental testing of fundamental theories of physics beyond the standard model and the exploration of cosmic phenomena through the techniques of particle physics. We are working on the MACRO experiment, which employs a large area underground detector to search for grand unification magnetic monopoles and dark matter candidates and to study cosmic ray muons as well as low and high energy neutrinos; the Chooz experiment to search for reactor neutrino oscillations at a distance of $1 \mathrm{~km}$ from the source; a new proposal (the Perry experiment) to construct a one kiloton liquid scintillator in the Fairport Ohio underground facility IMB to study neutrino oscillations with a $13 \mathrm{~km}$ baseline; and development of technology for improved liquid scintillators and for very low background materials in support of the MACRO and Perry experiments and for new solar neutrino experiments.
\end{abstract}




\section{Introduction}

This document is a progress report for the Drexel University-United States Department of Energy program of research in experimental particle physics.

The Drexel University group currently consists of one senior faculty member (R.I. Steinberg), one junior faculty member (C.E. Lane), two advanced graduate students (M. Mittelbrunn and F.C. Wang), a beginning graduate student (Z.L. Hu), an undergraduate physics major cooperative education assistant and an undergraduate physics senior project student.

Alșo working with the group is J. Petrakis, who did his doctoral thesis [1] on MACRO at Indiana University and who is currently a post-doctoral fellow at the nearby Bartol Research Foundation. Dr. Petrakis spends about $25 \%$ of his effort working with our group on physics analysis of data from the MACRO experiment.

We are presently active in the following research projects:

- the MACRO experiment, which employs a large area underground detector to search for grand unification magnetic monopoles and dark matter candidates and to study cosmic ray muons as well as atmospheric and stellar collapse neutrinos;

- a new one kilometer-baseline reactor neutrino experiment at the Chooz nuclear power station in France whose main goals are to seek neutrino vacuum oscillations down to a mass-difference squared sensitivity of $10^{-3} \mathrm{eV}^{2}$ (a ten-fold improvement over current limits) and to serve as a prototype for the Perry experiment;

- development of the Perry experiment, a $13 \mathrm{~km}$-baseline search for neutrino oscillations which will use a two-kiloton liquid scintillator and which is aimed at a mass-difference squared sensitivity of $8 \times 10^{-5} \mathrm{eV}^{2}$. The Perry experiment would close the gap between existing reactor neutrino oscillation searches and those using solar neutrinos. Perry would also be capable of precise measurement of the energy spectrum of ${ }^{8} \mathrm{~B}$ solar neutrinos down to an energy of $3 \mathrm{MeV}$, which would provide a new window on possible MSW oscillations of solar neutrinos;

- development of technology for improved liquid scintillators and for very low background materials in support of the MACRO, Chooz, Perry and other experiments; and 
- SSC research and development, particularly as applied to the GEM detector and to forward calorimetry.

MACRO - As described in Section 2, construction of the MACRO experiment in Hall B at the Gran Sasso underground laboratory (Abruzzi, Italy) is nearly complete. A substantial data set is already available from which a significant number of new physics results has been obtained and published; large quantities of excellent new data are anticipated after completion of the instrument.

Several million muons have been recorded by MACRO, and an extensive analysis of the early (1989-90) runs has been especially fruitful. Preliminary results were obtained on the vertical muon flux, the lateral spread and multiplicity distributions of muon bundles, a search for Grand Unified Theory magnetic monopoles, and a search for electron antineutrinos from stellar collapses, as well as surface-underground coincidences between MACRO and EASTOP (an extensive air shower array located on the mountain above MACRO and operated by a group from Turin University).

Recent publications of MACRO results concern gravitational stellar collapse [2], arrival time distributions of cosmic-ray muons [3], primary cosmicray composition [4], a search for strange quark matter [5], and a general description of the first supermodule.[6]

All six supermodules of MACRO were brought on-line this year, and the first supermodule has been retrofitted with new PMT's and electronics. New instrumentation being installed on MACRO includes the fast monopole and global muon trigger system, developed and constructed at Drexel by Prof. Lane and co-workers. This trigger system gives MACRO sensitivity to monopoles with velocity $\beta>10^{-3}$, and is important for fractionally charged particle searches as well. Installation of the Drexel fast monopole trigger system will be completed in the next few months.

The outstanding performance of the MACRO experiment has been made possible by the development of high transparency liquid scintillators in Prof. Steinberg's laboratory at Drexel University.

At present, more than 500,000 liters of the Drexel MACRO scintillator have been installed in the detector. The scintillator performance has been 
fully consistent with that obtained during development testing at Drexel. The attenuation length (the most critical parameter for the twelve-meter MACRO detector modules) is about $12 \mathrm{~m}$, including losses both from the liquid scintillator itself and from the totally reflecting teflon liner of the detector boxes. This high transparency means that the ratio of light collected from the near end of a module to that from the far end is only about $4: 1$. At the center of a tank, a minimum ionizing particle produces approximately 400 photoelectrons, while at a distance of 11 meters, such a particle yields 260 photoelectrons. No other detector of similar geometry has attained this level of performance.

In summary, our contributions to and responsibilities on MACRO are substantial. Beyond the continuing work of producing and testing the MACRO scintillator, we have responsibility for one of the major detector trigger systems and wish to participate as well in physics analysis of the data stream.

Chooz neutrino oscillation experiment - For our second major experiment, we have joined two French laboratories (the Collège de France, Paris and L.A.P.P. - Annecy), a Canadian group (Queen's University) and the University of New Mexiro to perform a sensitive new search for neutrino vacuum oscillations at a 125 meter-deep underground site in northeastern France $1 \mathrm{~km}$ from the nearly-completed Chooz nuclear power station (the Chooz experiment, Section 4). We are fortunate here to be able to pursue a ten-fold improvement in the neutrino oscillation mass parameter, $\Delta m^{2}$, at modest cost.

The Perry experiment - In addition, we are vigorously pursuing a major new highly sensitive long baseline deep-underground reactor neutrino oscillation experiment (the Perry experiment, Section 5). This experiment will take advantage of the existing IMB Fairport (Ohio) underground facility and will use antineutrinos from the $3600 \mathrm{MW}$ Perry Nuclear Power Reactor, optimally situated at a distance of $12.9 \mathrm{~km}$ from the underground laboratory.

The goal of the Perry experiment is to improve by a factor of 200 current limits on the neutrino oscillation mass parameter, $\Delta m^{2}$, thereby providing a sensitive new probe for finite neutrino masses. For large mixing angles, the experiment will detect oscillations for values of $\Delta m^{2}>8 \times 10^{-5} \mathrm{eV}^{2}$. This result would close the gap between current laboratory oscillation limits 
and the region accessible only via solar neutrino experiments. In addition, it would confirm or eliminate $\nu_{\mu}-\nu_{e}$ oscillations as the source of the atmospheric neutrino anomaly.[7]

The concept of the experiment is to build a 16-m-diameter cylindrical stainless steel tank in the old IMB site. The tank will be filled with two metric kilotons (one kiloton fiducial) of a highly transparent gadoliniumloaded liquid scintillator under development at Drexel.

Scintillation photons from particle interactions in the vessel will be collected by 2400 eight-inch photomultipliers and processed by fast multi-hit adc/tdc's. The Perry detector will have good energy resolution, with about 100 photoelectrons detected per $\mathrm{MeV}$ of ionization energy deposited.

About 4000 tons of ultra-pure water instrumented with 400 photomultiplier tubes will surround the scintillation tank, serving both as passive shielding against ambient radioactivity and as active shielding against cosmic ray muon-induced backgrounds, already suppressed by a factor of $2 \times 10^{4}$ by the 550 -meter rock overburden. Development work aimed at the submission of a formal proposal by the middle of 1993 is in progress.

Fairport Laboratory Workshop - Nearly 40 physicists from 11 institutions and 3 countries attended the Workshop on Future Experiments at the IMB Fairport Underground Laboratory, which we organized recently in collaboration with the University of New Mexico. The excellent turnout attested to the high community interest in new underground experiments, especially those directed at a new long baseline neutrino oscillation experiments, such as Chooz and Perry.

The dual purposes of the Workshop were to review neutrino oscillations, dark matter searches and closely relaied issues; and to develop a plan for the pursuit of new experiments in these areas.

The theoretical and experimental talks at the Workshop clearly showed that the question of neutrino masses remains at the very core of modern particle physics. The puzzle of the missing solar neutrinos may be explained by the unexpected existence of small but definitely non-zero neutrino masses. If confirmed by new experiments, the finite neutrino masses are likely to become the first phenomena beyond the bounds of the standard model of particle physics, and hence the key to a new paradigm for the field - perhaps a grand unified theory or a supersymmetric theory. 
The outcome of this workshop was the formation of a new collaboration to study, propose, and ultimately to perform the Perry experiment. Drexel's Prof. Steinberg was selected as spokesman for the new collaboration.

Collaborating institutions so far are the California Institute of Technology, Cleveland State University, Collège de France, Drexel University, L.A.P.P. (Annecy), Louisiana State University, Queen's University, University of Arizona, University of California at Irvine (observer status), University of New Mexico, and Vanderbilt University. Discussions are currently in progress with a number of others interested in joining the collaboration.

SSC - We have been active as well in developing liquid scintillators for use in SSC forward calorimeters (Section 6). While this is a small project, the materials and performance criteria have quite a large overlap with our other projects using liquid scintillator.

This progress report concludes with a list of our recent publications (Section 7). 


\section{The MACRO Experiment}

The MACRO experiment [8] (see Fig. 1) is a joint U.S.-Italian effort to construct and operate a magnetic monopole and cosmic ray muon and neutrino detector of unprecedented sophistication and sensitivity. The construction of the lower part of MACRO, which consists of six 'supermodules' $(12 \mathrm{~m} \times 12 \mathrm{~m} \times 4.8 \mathrm{~m}$ each), was completed in the Spring of 1991, with instrumentation of these supermodules currently underway. We started physics runs with all six supermodules in early 1992 .

Construction of the upper part of MACRO (the attico) was begun in the past year. When the attico is completed, MACRO will have dimensions of $72 \mathrm{~m} \times 12 \mathrm{~m} \times 9 \mathrm{~m}$, with an acceptance for isotropic particle fluxes of $10,000 \mathrm{~m}^{2} \mathrm{sr}$.

\subsection{MACRO Liquid Scintillator}

Full implementation of the MACRO detector will require a total of almost one million liters of liquid scintillator. Since no commercial scintillator was available with sufficient transparency to give satisfactory performance in a detector such as MACRO with its twelve-meter light collection path, our group took the responsibility of developing for MACRO a special high-transparency liquid scintillator.

\subsubsection{Design}

As a result of research in the scintillation la' 'oratory at Drexel University, we succeeded in designing a new liquid scintillator (now widely known as 'MACRO scintillator') with outstanding transparency (greater than twelve meters attenuation length for scintillation light) and good energy conversion efficiency (about $40 \%$ that of anthracene, the most efficient known organic scintillator). Furthermore, in collaboration with our MACRO colleagues at Indiana University and the University of Michigan, we have produced the MACRO scintillator in large quantities at a cost of about $\$ 1.25 /$ liter. For comparison, commercial liquid scintillators, even in large quantities, have been quoted to us at prices exceeding $\$ 2.00 /$ liter. We are therefore providing for MACRO a superior product at a saving of $\$ .75 /$ liter. For the million-liter finished detector, the total saving will be about $\$ 750 \mathrm{~K}$. 


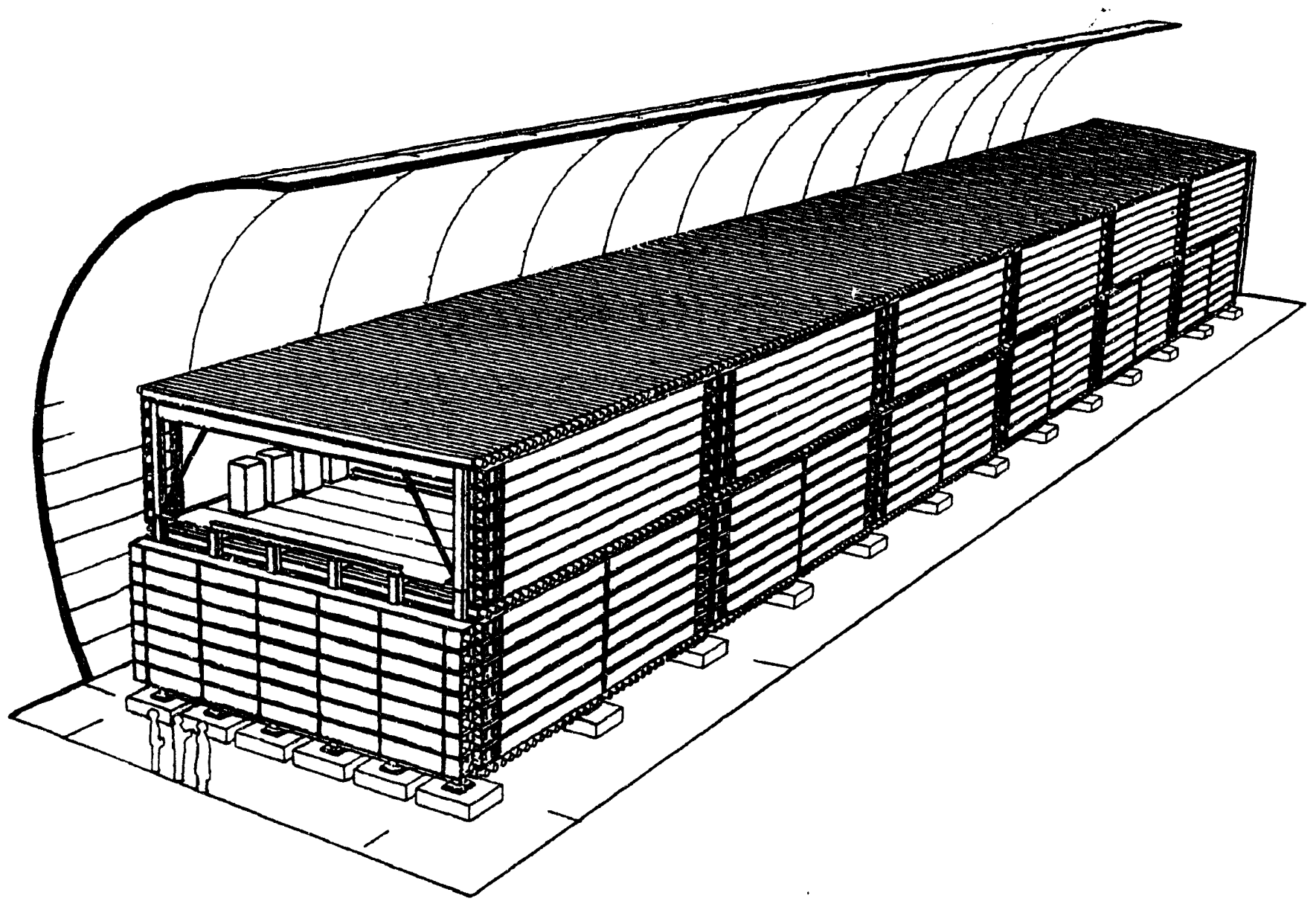

Figure 1: Schematic view of the MACRO experiment. The detector area of about $1000 \mathrm{~m}^{2}$ will allow a search for magnetic monopoles at a sensitivity level well below the Parker limit as well as significant new experiments in cosmic ray muon and neutrino physics. The principal U.S. contribution to MACRO consists of 500 twelve-meter long scintillation detectors and associated electronics. Nearly one million liters of the Drexel liquid scintillator are being produced for MACRO. 


\subsubsection{Production and Testing}

The process of producing the MACRO scintillator begins with preparation of scintillator concentrate at Drexel, together with direct shipment of previously tested and approved pseudocumene and mineral oil from their respective refineries to a liquid scintillator storage and blending facility at the Frascati laboratory near Rome, where the final mixing of the components is performed. The finished liquid scintillator is then transported to the Gran Sasso laboratory for installation in the MACRO detector.

This procedure allows the most critical steps to take place in the U.S. under the direct supervision of physicists. Testing of all ingredients (mineral oil, pseudocumene, PPO, bis-MSB, and stabilizers) and shipping containers (drums, Iso-tanks, etc.) and the critical step of producing the liquid scintillator concentrate therefore all take place with good quality control.

The extra shipping costs associated with scintillator concentrate production at Drexel are negligible compared with savings resulting from decreased risk of using substandard ingredients, contamination, error, or ot her failure. Only the scintillator concentrate ingredients $(<1 \%$ of the finished scintillator) are subject to the extra step of transport from the various manufacturers to our mixing facility in Philadelphia before being shipped on to Italy.

At Drexel University we have currently produced 4,400 liters of liquid scintillator concentrate. The concentrate is shipped in nitrogen-blanketed drums from Philadelphia to Italy. In addition, we have provided 22,000 liters of pure scintillation grade pseudocumene for the experiment. These ingredients, when mixed with mineral oil, are sufficient for 730,000 liters of finished scintillator. As in prior years, the mineral oil we are using was produced from Alberta crude oil drawn from the Trans-Canada pipeline, refined in Perth Amboy, New Jersey and shipped in containerized 20,000liter Iso-tanks to Frascati.

Rigorous quality control tests at each step in the preparation of the MACRO scintillator assure that all components meet our specifications. The quality control tests consist of analysis of pre-shipment samples from each batch of mineral oil, pseudocumene, and scintillation fluors. The entire production operation is proceeding smoothly, on time and under budget, and includes stringent personnel and fire safety precautions. 


\subsection{MACRO Fast Monopole Electronics}

\subsubsection{Introduction}

Magnetic monopoles are predicted in many varieties of Grand Unified Theories (GUT's). The GUT monopole should be supermassive $\left(M \sim 10^{16} \mathrm{GeV}\right)$ and would be found only in cosmic rays as a remnant of the high-temperature processes in the early universe.

One of the major objectives of the MACRO experiment is to detect GUT monopoles, or to set limits on them at a level that is low enough to exclude monopoles as a significant fraction of the 'missing mass' of the universe.

The Particle Physics group at Drexel is implementing the Fast Monopole (FASTM) trigger for MACRO, with the goal of detecting monopoles with velocities $\beta \gtrsim 2.5 \times 10^{-3}$. The FASTM trigger is implemented jointly with a 'global' fast particle trigger, since most of the triggering electronics is the same.

\subsubsection{MACRO Fast Monopole Physics}

In the next few years, MACRO will be able to set new limits on magnetic monopole fluxes well below the Parker bound [9] for monopoles with $10^{-4}<$ $\beta<1$ (see Fig. 2). Current limits on 'fast' monopoles $\left(\beta \geq 10^{-2}\right)$ are already below the Parker bound; however, MACRO should be able to place limits at least a factor of ten below other monopole search limits over a broad range of monopole velocities.

The ability to detect GUT magnetic monopoles in MACRO hinges upon the scintillation light yield of a monopole passing through MACRO's liquid scintillation counters. This light yield has been calculated using experimental data for the scintillation yield of slow protons $[10,11]$, and is shown as a function of monopole velocity $\beta$ in Fig. 3.

Since monopoles are expected to be traveling at $\beta<1$, this scintillation light is spread out over the transit time of the monopole through the counter, and gives a distinctive 'square' experimental signature (see Fig. 4). Fast monopoles have a less distinctive pulse, since the transit time through the scintillation counters is much less than that of slow monopoles. The time difference between the signals from monopole entry and exit, however, is still a very useful experimental handle. 




Figure 2: Astrophysical and experimental bounds (1989) on the flux of GUT monopoles.

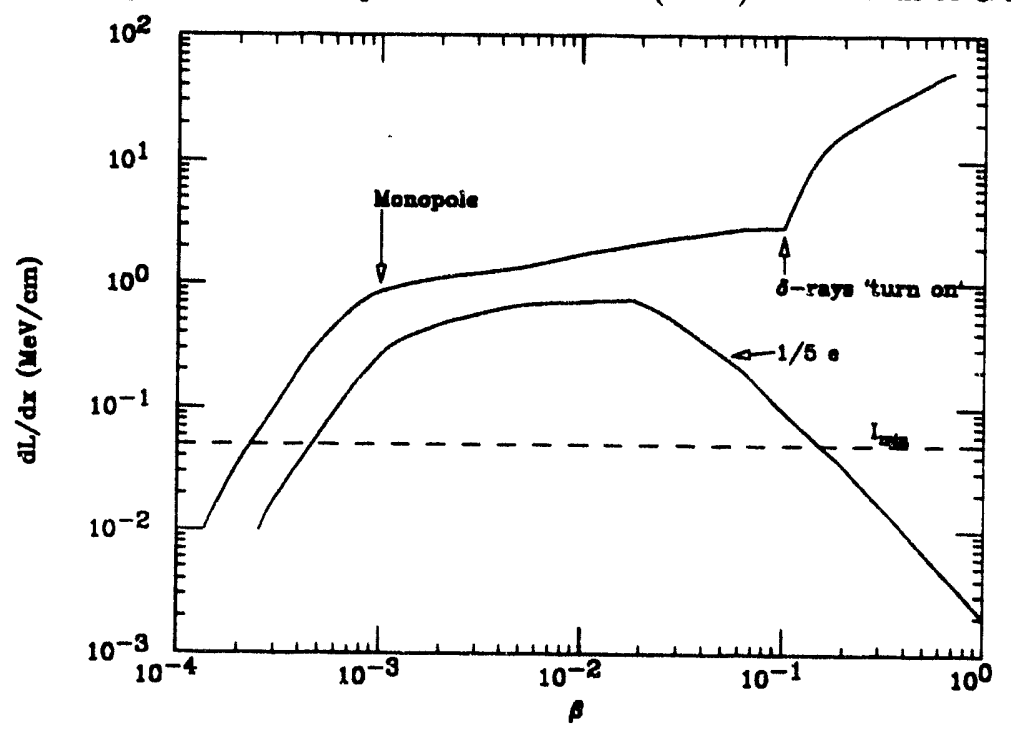

Figure 3: Expected scintillation yield, $d L / d x$, for a magnetic monopole and for a charge $1 / 5$ e particle compared to that of a minimum ionizing particle, $I_{\min }$. The efficiency of the scintillator for converting ionization energy to light is $2.5 \%$. A minimum ionizing particle whose ionization rate is $2 \mathrm{MeV} / \mathrm{cm}$ therefore has a scintillation yield of $.05 \mathrm{MeV} / \mathrm{cm}$. 


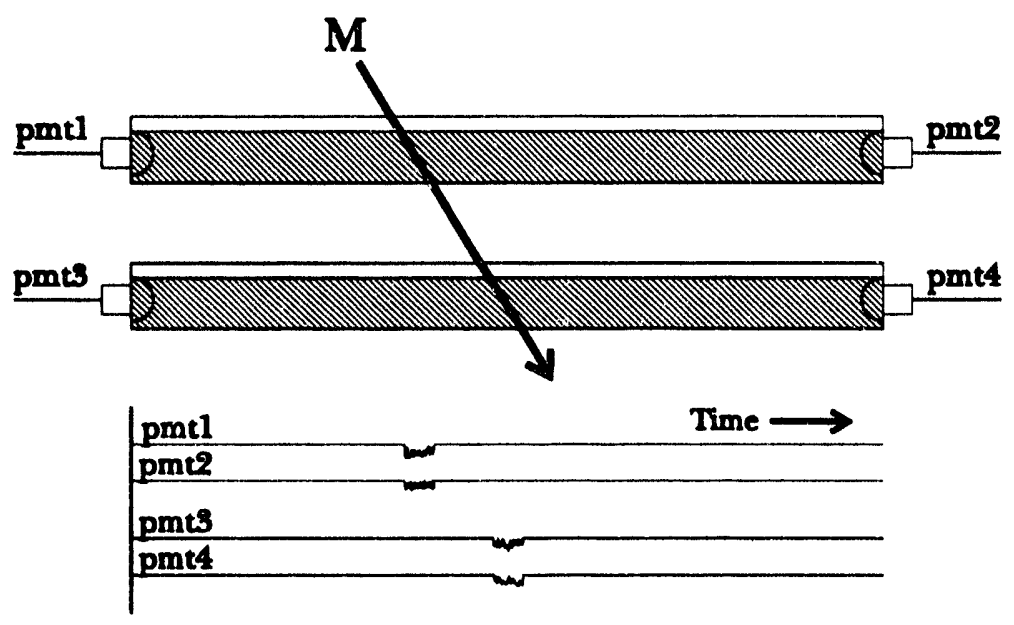

Figure 4: Experimental signature for a monopole passing through two layers of MACRO. The pulse widths are from the finite module transit time of the monopole.

It should be noted that while the time and pulse-shape signatures of monopoles distinguish them from cosmic rays and other backgrounds, there are many species of proposed massive particles that could produce a similar signature. This possibility is less a problem than an opportunity, since the detection of any such massive particle would signal new physics at very high energy scales.

\subsubsection{Trigger Design}

Unlike triggers for 'slow' monopoles $\left(\beta<10^{-3}\right)$, it is not necessary to have very low thresholds to be sensitive to fast monopoles. At the same time, the fast monopoles transit a scintillation counter quickly enough that the pulse shape from the PMT's does not give a very good means of distinguishing fast monopole candidates from cosmic ray muons at the trigger level (see Fig. 5). Signals from muons or radioactive decays very near a PMT that saturate the PMT and give 'square' pulses can mimic the pulse shape of a fast monopole. It is unlikely, however, that background can give a particle track with $\beta$ significantly less than one. 


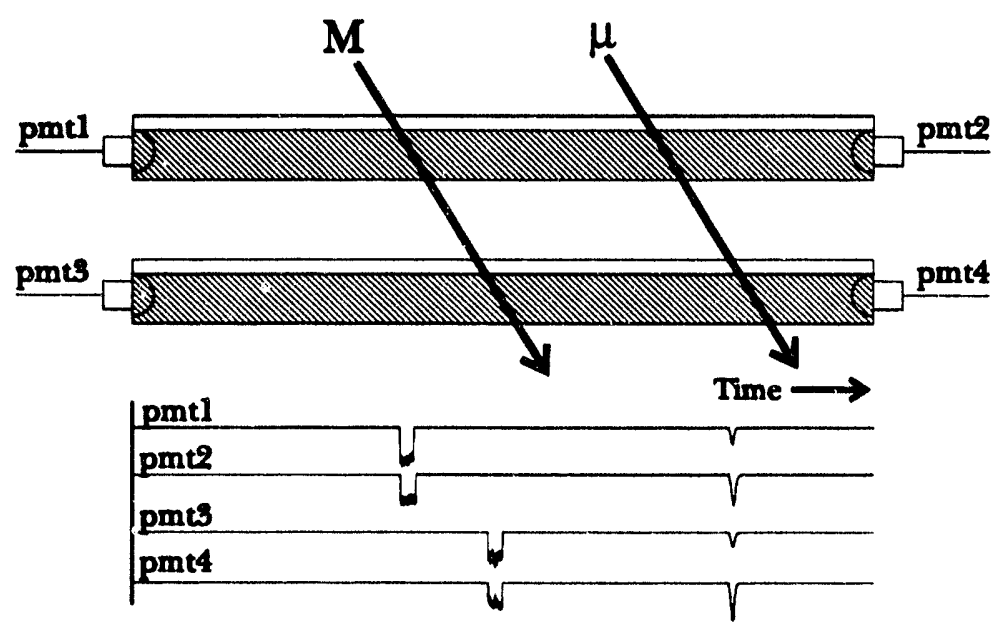

Figure 5: Comparison of signatures in MACRO for cosmic-ray muons and fast monopoles.

The main difficulty for a fast monopole trigger is the copious flux of cosmic ray muons with $\beta=1$. A combination of pulse amplitude and timing discrimination is required to reject muons with the necessary efficiency. This rejection cannot he done on a 'single counter' basis, however, since one of the primary signatures for a muon is its time-of-flight between MACRO counters. Instead, we will use a delayed coincidence between different planes of scintillation counters to reject prompt $(\beta=1)$ background. Some geometric inefficiency thereby will necessarily be introduced due to cracks and dead spaces in MACRO, and because of the need to limit the number of counters that can participate in the trigger.

For the scheme described below with an isotropic flux of fast monopoles, we have calculated that there is a $\approx 15 \%$ geometric inefficiency, mostly due to 'corner clippers' that have very short transit times through MACRO. A further stage of data analysis of muon events may be able to recover some of the efficiency lost to such geometric effects.

In five years of running with six supermodules, the number of cosmic 
ray muons expected (scaling from the Spring ' 89 run data) is about $3 \times 10^{7}$. During that same time, one might expect a few fast monopole candidates.

Because of the very low flux limits that MACRO will be able to probe, it is possible that there is indeed a detectable monopole flux. This possibility mandates that monopole triggers cause an extensive readout of the detector, since each trigger could be a long-awaited monopole going through the detector.

The extensive readout makes it very expensive in terms of deadtime and data rate to produce false monopole triggers, and puts a premium on the rejection capabilities of the monopole trigger logic. Not only must the triggers be efficient, but they need to be noise free.

The trigger design that will be implemented for MACRO consists of three 'subtriggers', to cover the area of interest in the $d L / d x$ vs $\beta$ plane while efficiently rejecting cosmic-ray muons. Such a design will give MACRO full sensitivity to fast monopoles as well as sensitivity to massive fractionally charged particles (such as the $1 / 5 e$ particles suggested in some string theories [12]), together with an active muon veto that can be used as a prompt muon trigger in global muon trigger logic.

The $\mu$ trigger is a fast coincidence of scintillator planes, suitable for use as a global muon trigger and as a muon veto for monopole triggers. The FASTM trigger is a delayed coincidence of scintillator planes, with a veto from the $\mu$ trigger to exclude muons. The highly ionizing particle trigger (HIPT) is also a delayed coincidence of scintillator planes, with a higher threshold.

The trigger logic (see Fig. 6) uses signals from fanned-in sets of 8 scintillation counters. The combined signals are discriminated, and a coincidence of iswo ends of the set of scintillation counters is required within a short time window together with a coincidence of two 'planes' of MACRO within a longer time window.

Last year we developed and put into limited production an 8:1 linear fanin with coaxial ribbon cable input having normal and fast-integrated signal outputs. Discriminating on a signal that is integrated with a time-constant of $\sim 50 \mathrm{~ns}$ gives a slower drop-off of discriminator sensitivity as a function of monopole velocity, which is desirable for fast monopole detection.

Fig. 7 shows the regions in $\beta$ and $d L / d x$ which are covered by the FASTM, HIFT and $\mu$ veto triggers. The FASTM trigger is vetoed by the $\mu$ trigger to prevent muons from swamping the fast monopole triggers. HIPT trigger levels are set at $\sim 10 \times I_{\min }$ which should be sensitive to very fast monopoles, 


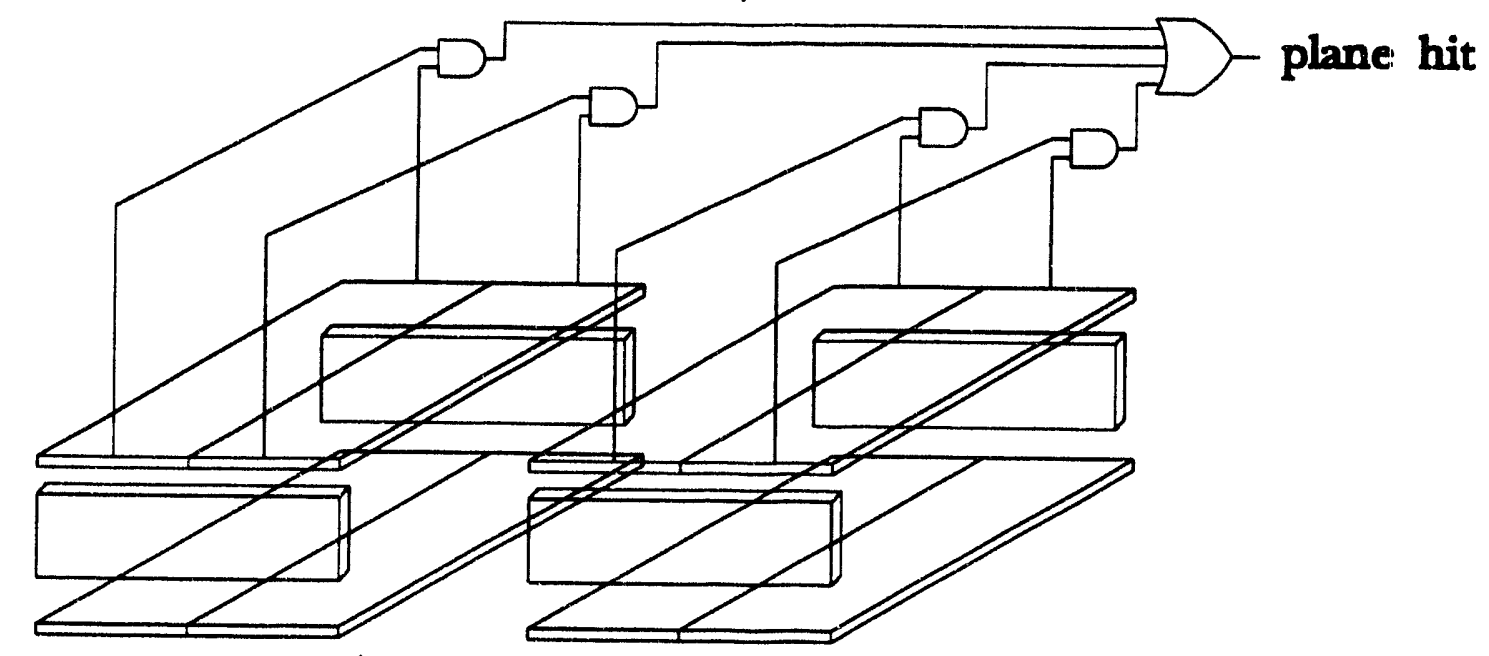

Supermodule 1

Supermodule 2

Figure 6: The fast monopole trigger logic uses an end-to-end coincidence of signals from a set of 8 scintillation counters, and combines these coincidence signals to make a 'plane hit' signal. Two or more planes being hit within a coincidence interval produces a trigger.

yet have a low false trigger rate from muon events. Saturation effects in the PMT's can make for difficulties in implementing a high-threshold trigger; the saturation characteristics of the PMT's is a subject of active study at this time.

These triggers will be combined for adjacent overlapping pairs of supermodules to provide more complete angular coverage for fast monopoles. If singles rates allow, this combination of triggers can be extended to include more supermodules in an individual triggering group. 


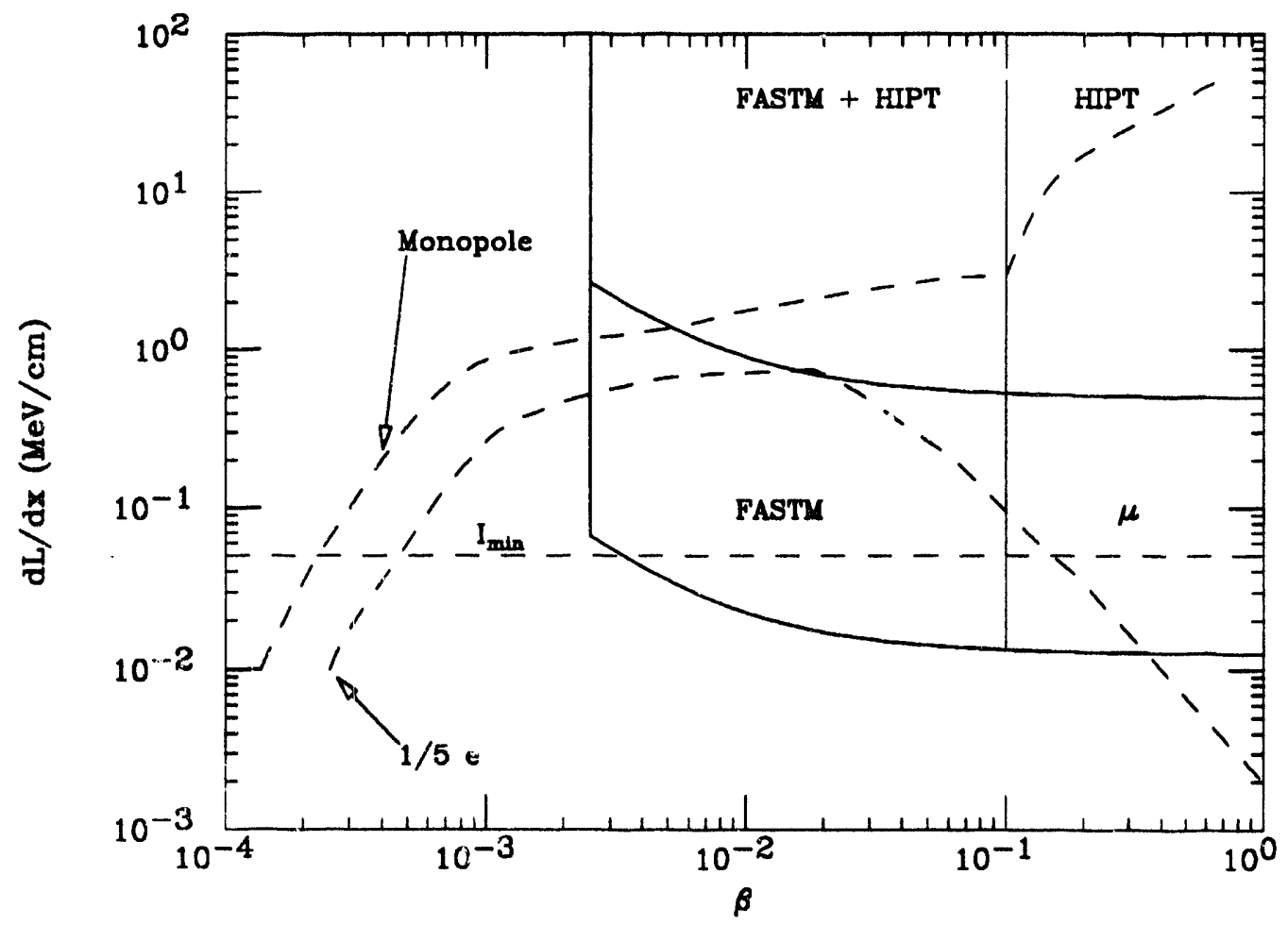

Figure 7: Coverage of the FASTM, Highly Ionizing Particle (HIPT) and $\mu$ veto triggers in $d L / d x$ vs $\beta$. The triggers are effective for GUT monopoles with $\beta>2.5 \times 10^{-3}$ and for $1 / 5 e$ particles with $2.5 \times 10^{-3}<\beta<0.1$. For explanation of $d L / d x$ see Fig. 3 caption. 


\subsubsection{Implementation}

Overview Achieving the capability required of the fast monopole trigger within a limited budget has required us to design some custom electronics. The fast monopole trigger operates with highly fanned-in signals to reduce the channel count of trigger electronics. Even so, the number of channels needed for MACRO is quite large.

Signals from the PMT's are fanned out to a number of trigger and acquisition systems; the signals for the fast monopole trigger are sent through a splitter printed circuit board (PCB) to obtain the correct combination of signals on each cable, then linearly added to make a 'patch end' signal for 8 scintillator tanks.

The patch ends are discriminated at $\approx 100 \mathrm{mV}$ in a commercial programmable high-density discriminator; an end-to-end fast coincidence is then required of the discriminated signals to make a 'patch hit'. Up to four adjacent patches on the same plane of MACRO are then ORed to make a 'plane hit', and a 2 fold coincidence of plane hits is used to trigger the experiment.

The custom components of this system are the splitters, fanins, and plane logic (FMT modules) for patch-end AND/ORing.

Linear Fanins The first challenge for a linear fanin was in achieving an 8-to-1 analog fanin of PMT signals with sufficient dynamic range for fast monopole signals. Commercial fanins have strictly limited range (typically $1 \mathrm{~V}$ ), and the most attractive possibility for a commercial $8: 1$ fanin is no longer in production.

As a result, it was necessary for us to design and build a fast analog 8:1 fanin. The fanin was developed and tested in prototype form in the summer of 1990; a CAD (computer automated design) program was used to generate a custom PCB layout for commercial board production.

A sufficient number of fanin modules have been assembled and tested to implement the fast monopole trigger on all of MACRO.

Fig. 8 shows the linear fanin design. Major criteria for this fanin are linearity for small pulses and a large dynamic range, since it is expected that fast monopoles will produce very large PMT pulses. The fanin schematic shown has a $6 \mathrm{~V}$ input dynamic range, a gain of about 0.66 in its linear range, and a PSRR (power supply rejection ratio) of less than $0.1 \%$.

Both the linearity and the dynamic range of the fanin design are slightly 


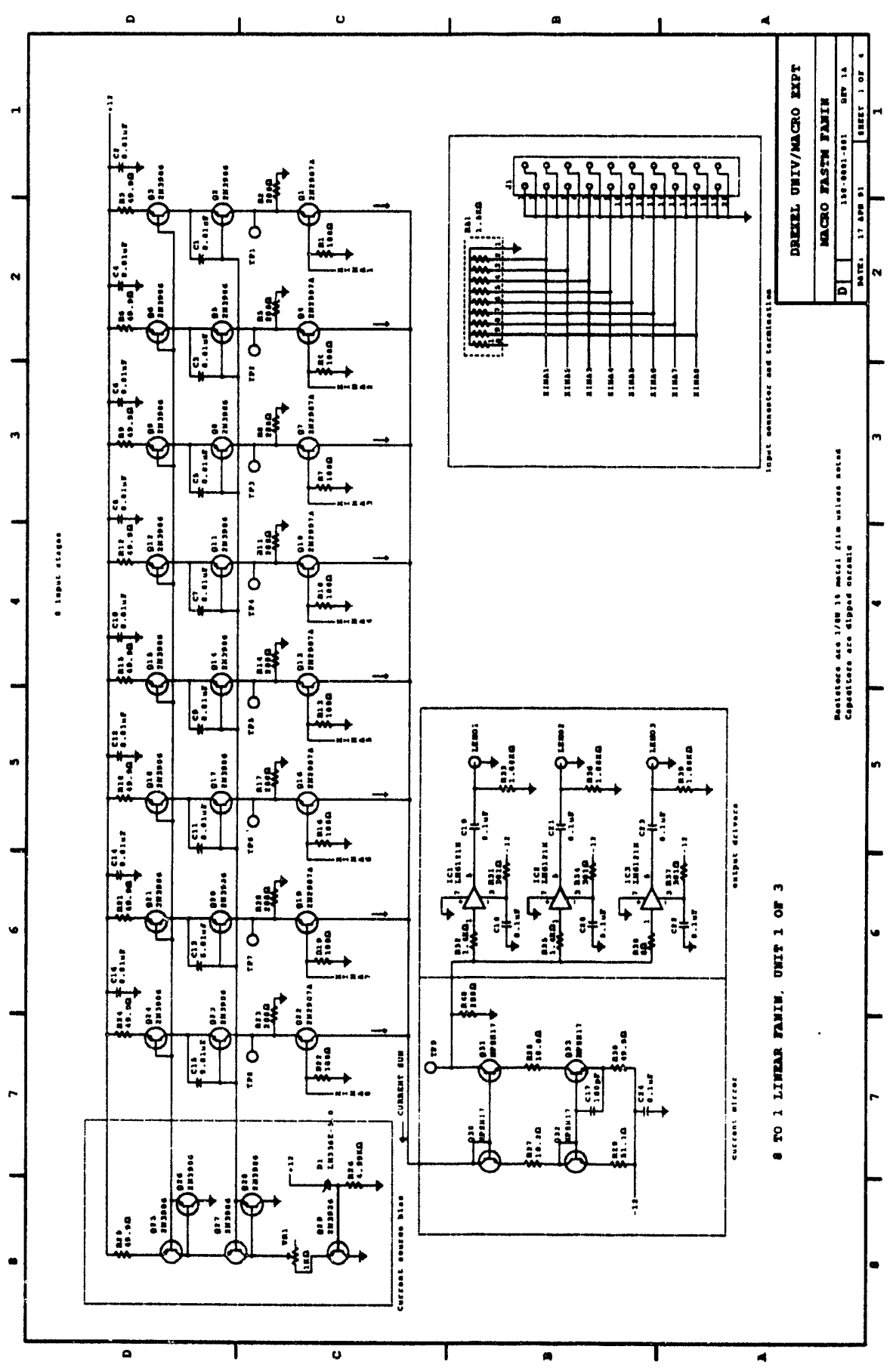

Figure 8: Schematic for the fast linear 8:1 fanin. Current from each input stage is summed at the input of a current-mirror. Two of the fanin outputs are integrated with time constants of $\approx 50 \mathrm{~ns}$. 


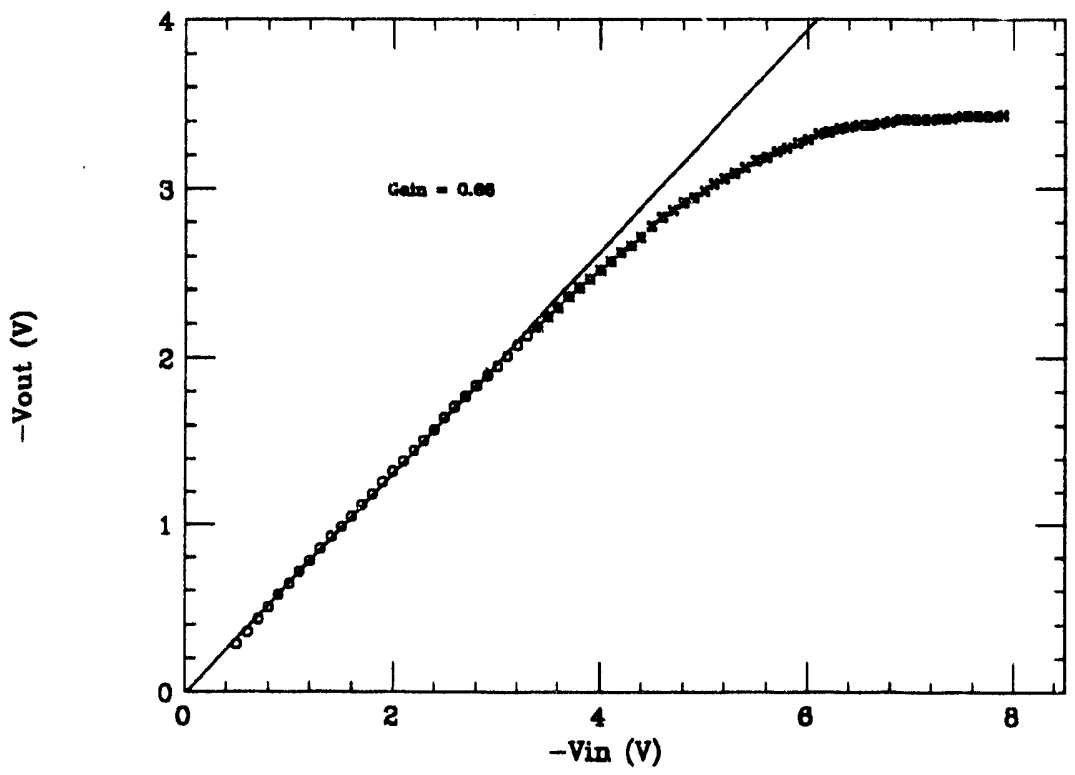

Figure 9: Response function of production fanin, showing linearity up to $\approx 3 \mathrm{~V}$, with saturation at an input of $\approx 6 \mathrm{~V}$.

less than achieved with early prototypes because of the need for added stability under a variety of load conditions. Fig. 9 shows the output voltage as a function of input voltage on one channel for one of the production fanins. The saturation shown in the figure is occurring on the input circuitry for the fanin; the output circuitry saturates at a higher level, which allows summation of saturated signals. Because the signals out of the fanin are being discriminated rather than measured for acquisition, the nonlinearity for large input signals is not expected to be problem.

The channel-to-channel variation of the fanin response functions is quite small, particularly in the linear response region. Fig. 10 shows response functions for all eight channels of a fanin. The variation between different fanin units is also quite small, allowing global thresholds to be used on fanned in signals without the need for individual adjustments.

The fanin is designed to be appropriate for implementation on a thick-film hybrid or on a bipolar chip; however, a discrete component implementation is used for MACRO because of the necessity for timely deployment. If further uses of this fanin are found, a more elegant packaging of the circuit will be investigated. 


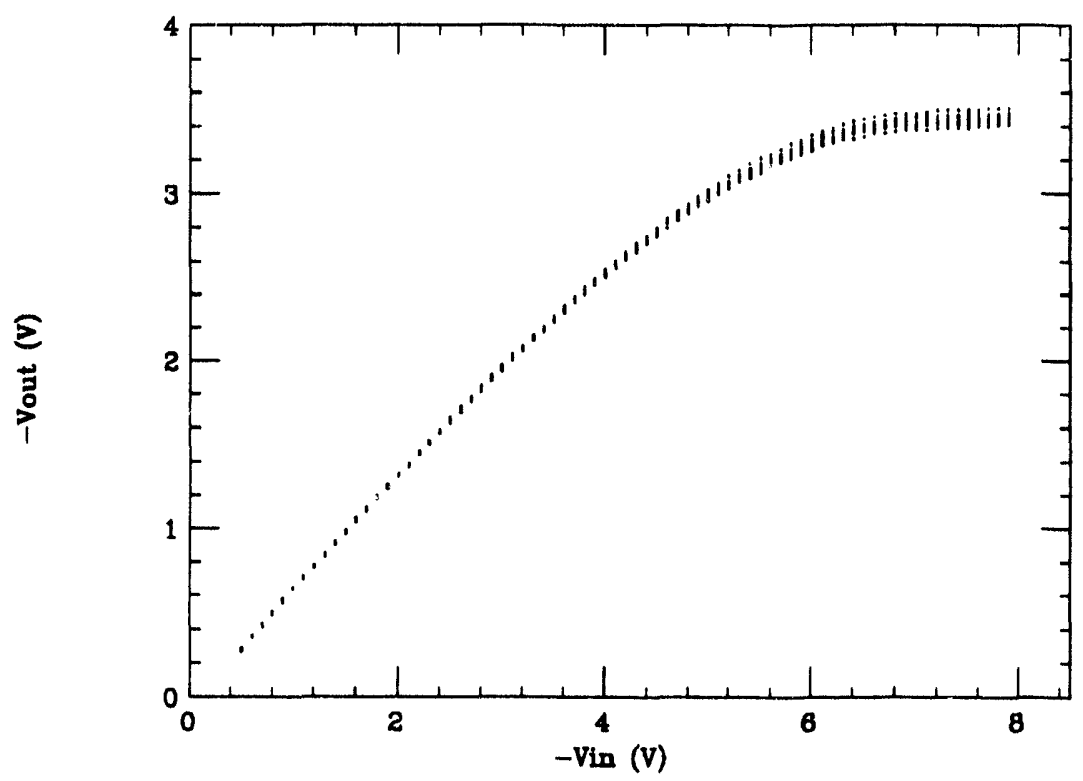

Figure 10: Response function of 8 inputs of fanin plotted together. Similar plots of different fanin units are nearly identical to this one.

Signal Splitter In addition, a 'signal splitter' PCB was designed and produced in quantity so that the signals available from the MACRO PMT fanouts could be recombined into the groups needed by the fanins.

Our cost studies show that close to half of the fanin cost is a 'per module' cost, regardless of how many functional units are contained in a module. By combining the signals appropriately, we can use a $50 \%$ higher circuit density on the fanin PCB's, and decrease the overall cost of the trigger.

The signal splitters take signals from two input cables in the form $1 R$, $1 \mathrm{~L}, 2 \mathrm{R}, 2 \mathrm{~L}, \ldots$ and route them to two output cables in the forms $1 \mathrm{R}, 2 \mathrm{R}$, ... and $1 \mathrm{~L}, 2 \mathrm{~L}, \ldots$, suitable for fanning in to make plane-end sums.

The trace widths required to maintain a constant characteristic impedance on the splitter PCB mandated the use of $93 \Omega$ coax ribbon cable for the inputs to the fanin, which also has the advantage of reducing the current load on the fanouts driving the ribbon cables. The fanin design allows a simple change of input characteristic impedance by changing a single resistor array on each input connector.

Six signal splitter PCB's are bolted to a rack-mount panel and provide signals for four 8:1 fanin modules. 
Fast Monopole Trigger (FMT) Module A prototype FMT module was designed and constructed in May 1991 for testing on MACRO. This module makes the fast coincidences of signals from opposite ends of a group of tanks, and also combines signals from the same plane of MACRO.

The final version of the boards for the FMT modules was received from the PCB manufaciurer in April 1992. Twenty boards have been stuffed and tested over the summer, and are being shipped to LNGS this Fall.

The most recent schematic for the FMT module (see fig. 11) includes a rate-monitoring capability, and a digital pulse-stretcher and latch for reading out trigger patterns. These new functions were easiest to implement in the FMT module because the necessary signals were already present in the module, and the coincidence logic leaves sufficient board space so that these extra functions can be easily implemented.

Cables A significant expense in the fast monopole trigger is the large number of ribbon coax cables used for primary signal inputs. After consultation with cable manufacturers and companies specializing in cable assembly, we found that it would be most cost-effective to purchase the cable-making equipment and to assemble the cables on-site to the necessary lengths. We expect the cable-making facilities to be generally useful in MACRO and other future experiments using many channels of analog information.

Recently we were informed of problems in the manufacture of ribbon coax cable, and as a result switched our new cabling from $93 \Omega$ to $75 \Omega$ in order to take delivery of existing stock. Measurements made with $50 \Omega$ cable indicate that reflections in the splitter board are not a problem as long as the fanins are terminated properly. The fanins that are being fed by the new cable are being modified by replacement of their termination resistor SIPs, and it is expected that there will be no problem with cable reflections.

Calibration The fast monopole trigger scheme has been developed in parallel with a scheme for calibration and monitoring of the detector and the trigger system. Since the flux of monopoles is extremely low, we lack a 'test beam' to see that our triggers are efficient. Our calibration and monitoring scheme uses a computer program (shown as a 'thought balloon' in Fig. 12) which generates an isotropic flux of fast monopoles through MACRO. The pathlengths, $d L / d x$, and scintillator response curves are factored in to give 


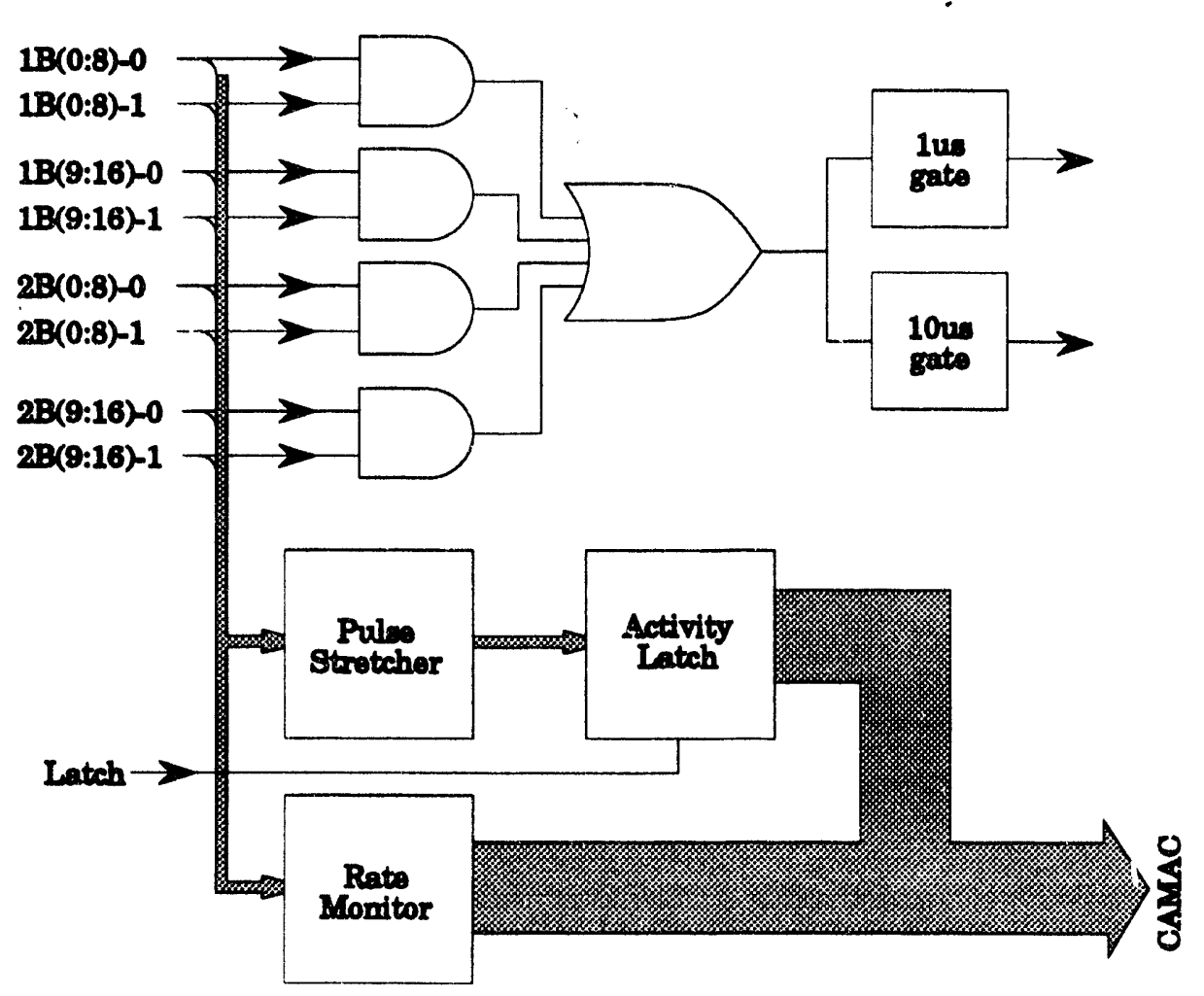

Figure 11: Block diagram of FMT module. The coincidence logic makes an end-to-end AND of patch-end signals, which are then ORed to give a plane hit signal. The FMT module also has a trigger pattern register, and rate-monitoring capability. 
the pulse height, width, and relative delay between PMT pulses in two scintillator counters (or two PMT's in bench-testing). The PMT response in photoelectrons is calibrated for given widths and pulse heights of LED driving pulses, and the intelligent pulse generator is given a set of instructions which result in a pair of pulses being sent out to the LED's.

Our calibration scheme thus produces PMT pulses which simulate a monopole's passage through MACRO, and can test the fast (and slow) monopole trigger efficiency for isotropic monopoles as a function of $\beta$, as well as testing for effects such as saturation of the PMT's or electronics with very large signal pulses.

This calibration system is being irnplemented jointly with the MACRO group at Boston University, which has responsibility for the development of relay modules for switching pulses to the appropriate MACRO scintillator tanks. 
$\therefore \quad$.

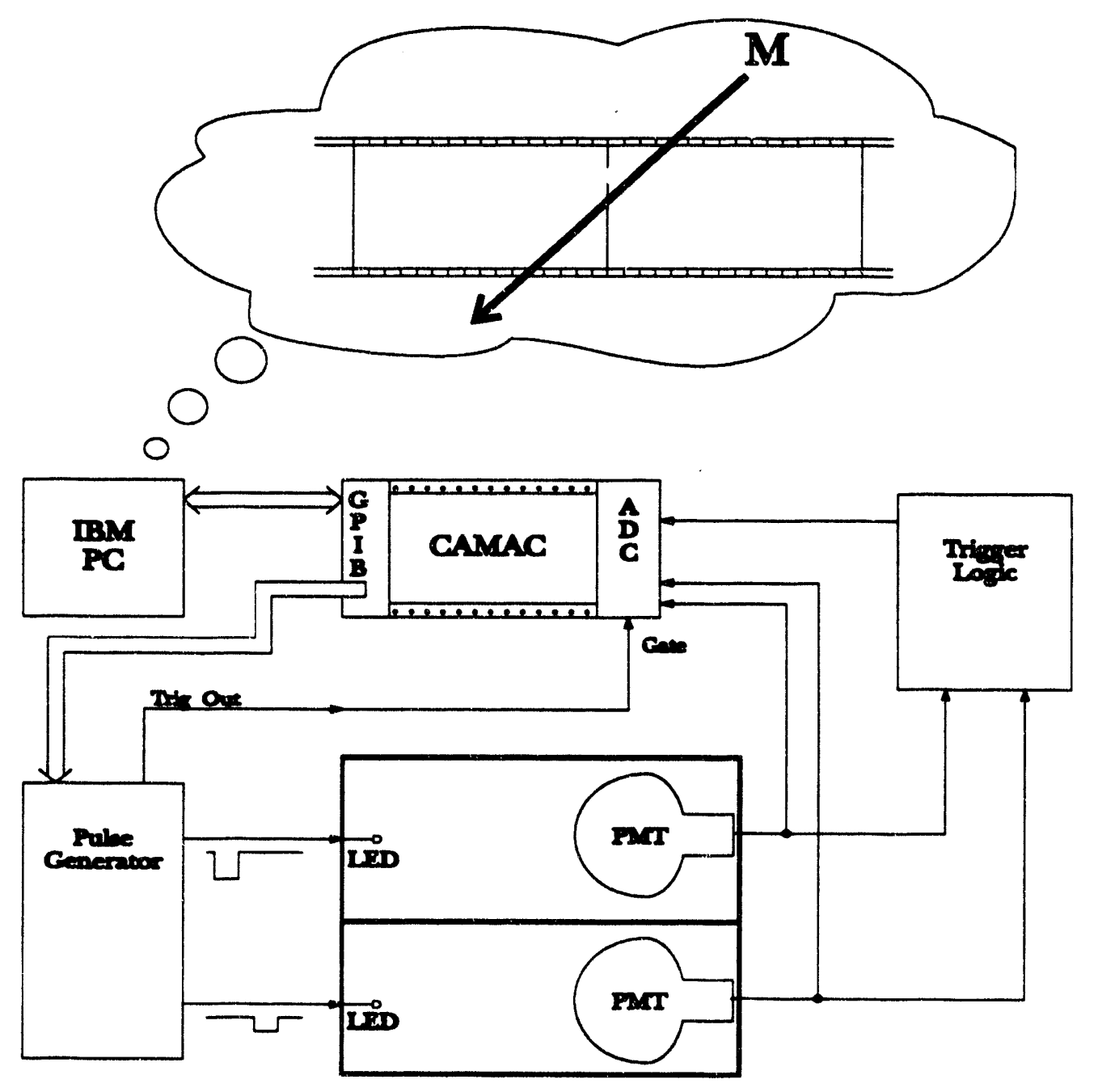

Figure 12: Calibration and testing scheme for fast monopole triggers. A program on the IBM-PC 'generates' an isotropic flux of fast monopoles, and fires calibrated LED pulses to simulate the monopoles in MACRO. This system can also be used with PMT's in dark boxes (as shown) to test the trigger logic at Drexel. 


\subsection{MACRO Data Analysis}

The calibration procedures for MACRO are currently under revision, with the primary goal being to improve the quality and completeness of the calibrations. This revision will require a closer connection between the calibration requirements for analysis and the calibration process.

We intend to automate the calibration as part of the online acquisition system (currently it is a standalone system), and to upgrade the calibration hardware to allow for more precise, higher statistics LED calibration runs. These improvements should benefit the monopole and neutrino burst triggers, since each of them depends critically on the setting of low-energy detection thresholds.

As described above (see Section 2.2.4), we are developing calibration and testing procedures for the monopole electronics. The first part of the calibration system was installed last year, and the remainder is expected in the next six months.

Our data analysis program is augmented by close collaboration with Dr. John Petrakis, who is devoting $25 \%$ of his time to MACRO data analysis. Dr. Petrakis is a postdoctoral fellow at the Bartol Institute, and brings with him a wealth of experience in MACRO data analysis. The analysis is currently focused on the search for delayed multi-muon events. The calibration and analysis techniques also have application to upgoing muon events from neutrino interactions, and exotic particle searches.

The past several years have seen an explosive growth in the quantity of MACRO data. The continuing strain of data tape copying, distribution and storage has pushed the collaboration to the use of compact $8 \mathrm{~mm}$ digital cartridge tapes as a storage medium. These tapes are now used for data distribution and much of the analysis of MACRO data. In normal running, MACRO now produces close to 1 GByte of event data each week.

In early 1991, we purchased an Exabyte $8 \mathrm{~mm}$ tape drive in order to analyze the current data tapes, and also upgraded the CPU of our MicroVAX to increase the CPU power available for analysis. The upgrade achieved a raw CPU performance increase of about 3.5 at modest cost. These upgrades have been accompanied by over 2 GByte of added disk space, to allow large data sets to be copied from tape and analyzed efficiently.

Our network connections (Bitnet, DECnet, and TCP/IP) allow us to exploit underutilized CPU resources at LNGS and elsewhere for analysis, 
but, clearly, more local computing power will be needed sometime in the next few years in order to keep the analysis effort from falling behind.

\subsection{Shifts and On-Site Work}

Construction and operation of the MACRO experiment require constant availability of significant manpower. To share the load equitably, a system of rotating shifts requiring each collaborator to work at the experimental site for two weeks once or twice per year has been set up. In addition, Prof. Lane and Michael Mittelbrunn are spending additional time at Gran Sasso for the installation of fast monopole trigger electronics. We expect that $\mathrm{Mr}$. Mittelbrunn will be resident at LNGS for most of 1994, as part of his work on a MACRO thesis. 


\section{$3 \quad$ Neutrino Oscillations}

\subsection{Introduction}

One of the central issues in particle physics, astrophysics and cosmology is the possible existence of a finite neutrino rest mass. In the minimal $S U(2) \otimes U(1)$ standard model of particle physics, the neutrinos are massless; however, this model, although successful wherever so-far tested, is a long way from being entirely satisfactory. Its predictions therefore deserve ever more rigorous experimental testing. Many extensions of the standard model are consistent with finite neutrino rest mass and many others require it.

Experimental hints of a finite neutrino mass exist both from the study of the flux of solar neutrinos (now determined to be deficient by four independent experiments) as well as from a possible anomaly in the flavor content of cosmic ray neutrino fluxes reported by several large underground detectors.

There is therefore excellent motivation for new experimental searches for a finite neutrino rest mass both from the theoretical and from the experimental point of view.

\subsection{Experimental Searches for Neutrino Mass}

There are a number of experimental approaches to detection of a finite neutrino rest mass. Precision measurements of low energy beta decay spectra in the vicinity of the end point (i.e. zero neutrino kinetic energy) offer perhaps the most direct approach. Current limits provided by this technique are in the vicinity of $m_{\bar{\nu}_{e}} \sim 10 \mathrm{eV}$.

Other methods sensitive to much smaller neutrino masses include observation of the time structure of neutrino pulses from collapsing stars and searches for neutrino matter and vacuum oscillations. Oscillation searches have the advantage of potentially exquisite sensitivity to the mass differences of the oscillating particles. For example, the observed strangeness oscillations in the $K^{0}-\bar{K}^{0}$ system reveal an underlying mass difference of $\Delta m=3.5 \times 10^{-6} \mathrm{eV}$ !

\subsection{Neutrino Vacuum Flavor Oscillations}

Only a single assumption, that of nonconservation of separate lepton number, is required to seek proof of finite neutrino mass by searching for neutrino 
vacuum oscillations. Since there is no compelling reason for believing in the separate and absolute conservation of $L_{e}, L_{\mu}$ and $L_{\tau}$, the existence of mass differences among the neutrino mass eigenstates $\left(\nu_{1}, \nu_{2}, \nu_{3}\right)$ would lead to their propagation with slightly different frequencies, as first envisioned by Maki [13] and by Pontecorvo [14].

Hence, an initially pure (i.e. an eigenstate of the weak interaction) beam of $\bar{\nu}_{e}$, which at the same time would be a coherent superposition of neutrino mass eigenstates, would develop at subsequent times a $\bar{\nu}_{\mu}$ component, while the $\bar{\nu}_{e}$ content of the propagating beam would undergo a corresponding decrease. An experimental measurement of the decrease of the $\bar{\nu}_{e}$ flux would therefore detect the presence of the neutrino oscillation and hence reveal the underlying finite neutrino rest mass. This type of measurement is known as a neutrino oscillation disappearance experiment and has the advantage of being sensitive to extremely small values of neutrino mass differences. In addition, disappearance experiments are sensitive to several reaction channels simultaneously. For example, $\bar{\nu}_{e}$ might oscillate into $\bar{\nu}_{\mu}$ or $\bar{\nu}_{\tau}$, or even into $\nu_{e}$.

Alternative methods of searching for neutrino vacuum oscillations by looking for the appearance of an otherwise absent neutrino flavor are also of interest and frequently offer the advantage of being sensitive to neutrino oscillations of rather small amplitude (i.e. having a small mixing angle, $\theta$ ). This type of experiment usually explores only a single reaction channel, which, depending on circumstances, could be either an advantage or a disadvantage.

\subsection{Reactor Neutrino Oscillation Experiments}

The sensitivity of a neutrino oscillation search by means of an appearance channel is governed in a two-flavor scenario by the equation for the probability $P\left(\nu_{e} \rightarrow \nu_{x}\right)$ that a neutrino of the first flavor will oscillate into a neutrino of the second flavor, e.g.

$$
P\left(\nu_{e} \rightarrow \nu_{x}\right)=\sin ^{2} 2 \theta \sin ^{2}\left(\frac{1.27 \Delta m^{2}\left[\mathrm{eV}^{2}\right] L[\mathrm{~m}]}{E_{\nu}[\mathrm{MeV}]}\right),
$$

where $\theta$ is the mixing angle, $\Delta m$ is the mass difference between the neutrino mass eigenstates, $L$ is the source-detector distance, and $E_{\nu}$ is the neutrino 
energy.

For a disappearance experiment, the relevant quantity is the probability $P\left(\nu_{e} \rightarrow \nu_{e}\right)=1-P\left(\nu_{e} \rightarrow \nu_{x}\right)$. In either case, the experimental sensitivity is determined by the ratio $L / E_{\nu}$. It is therefore important to use the largest value of $L$ consistent with adequate statistical information (hence a desire for long baseline experiments). At the same time, working at the lowest practical neutrino energy is also important.

Nuclear power reactors provide intense, rather well understood sources of low energy $\bar{\nu}_{e}$ 's. Over the years therefore, a number of neutrino oscillation searches have been performed at various reactors. A typical experiment of this type was performed at the Gösgen nuclear power reactor in Switzerland.[15]

The Gösgen experiment measured the $\bar{\nu}_{e}$ spectrum at distances up to 64.7 meters from the reactor by using the inverse beta decay reaction $\bar{\nu}_{e}+\mathrm{H} \rightarrow e^{+}+n$. The reactor had a thermal power of $2800 \mathrm{MW}$, while the detector provided a target mass of $320 \mathrm{~kg}$ and yielded a neutron detection efficiency of $21.7 \%$.

The results of neutrino oscillation experiments are often displayed on socalled exclusion plots, such as that shown in Fig. 13. In this plot, the area above and to the right of the curve labelled 'Gösgen' is the region of the $\Delta m^{2}$ $v s \sin ^{2} 2 \theta$ phase space where neutrino oscillations would have been detected by this experiment had they been present. The minimum detectable $\Delta m^{2}$ for maximal mixing was $1.9 \times 10^{-2} \mathrm{eV}^{2}$. 




Figure 13: Limits on neutrino vacuum oscillation parameters. 


\section{The Chooz Neutrino Oscillation Experi- ment}

\subsection{Introduction}

The Chooz experiment is a planned search for neutrino vacuum oscillations at a distance of one kilometer from a pair of large nuclear reactors nearing completion in northern France. An international consortium consisting of the Collège de France (Paris), LAPP (Annecy, France), Queen's University (Kingston, Ontario), the Kurchatov Insiitute (Moscow) and our Drexel University group has agreed to collaborate on this effort.

The neutrino source is a pair of reactors each of which will have a power of $4.2 \mathrm{GW}$-thermal. The two reactors are scheduled for startup at the end of 1995 and the middle of 1996, respectively. An essential feature of the experimental site is the availability of a tunnel with an overburden of 125 $\mathrm{m}$ of rock, equivalent to $300 \mathrm{~m}$ of water. Building the neutrino detector in this tunnel will provide the cosmic-ray shielding needed to preserve the signal/noise ratio against a one hundred-fold neutrino flux reduction with respect to previous experiments.

The experiment will look for the spectral distortion and flux reduction which would signal the existence of vacuum oscillations of the electron antineutrino beam. The limits on neutrino oscillations expected are shown in Fig. 13 by the curve labelled 'Chooz'. For maximal mixing, the experiment would detect oscillations for values of $\Delta m^{2}>1 \times 10^{-3} \mathrm{eV}^{2}$, an order-ofmagnitude improvement over the currently available limits.

The experiment would also provide an excellent testing ground for techniques and calculations needed for the larger Perry experiment (section 5).

\subsection{Overview}

The Chooz neutrino detector will consist of a $180-\mathrm{cm}$-diameter cylindrical steel tank shielded by a $150-\mathrm{cm}$-thick active veto water Čerenkov detector. The tank will be filled with seven tons of a special gadolinium-loaded liquid scintillator developed at Drexel University. Scintillation photons from parti- 
Table 1: Parameters of the Chooz Scintillation Detector

\begin{tabular}{|c|c|}
\hline \multicolumn{2}{|c|}{$\begin{array}{c}\text { CHOOZ DETECTOR } \\
\text { PARAMETERS }\end{array}$} \\
\hline Scintillator height, cm (cyl) & 243.8 \\
\hline Scintillator radius, cm (cyl) & 91.4 \\
\hline Scintillator volume, m3 & 6.4 \\
\hline Number of PMT's & 46 \\
\hline PMT radius, cm & 6.35 \\
\hline PMT direct coverage & $3.0 \%$ \\
\hline Reflector enhancement & 3.0 \\
\hline Scintillation yield (\% anthr) & $45 \%$ \\
\hline oV per scintillation photon & 151 \\
\hline QE $\times$ collection factor & 0.14 \\
\hline Atrenuation length, $m$ & 8 \\
\hline Typical light attenuation & 0.89 \\
\hline Photoelectrons/MeV & 75 \\
\hline
\end{tabular}

cle interactions in the vessel will be collected by 46 five-inch photomultipliers and processed by fast multi-hit adc/tdc's. The detector will have good energy resolution, with about 75 photoelectrons detected per $\mathrm{MeV}$ of ionization energy deposited. The parameters of the detector are shown in Table 1.

Primary shielding against background is provided by the 125-meter thick rock overburden, which reduces the surface cosmic ray muon flux by a factor of 200, as shown in Fig. 14, a muon depth-intensity plot. The residual cosmic ray background is further suppressed by active shielding consisting of a water Cerenkov veto detector surrounding the steel scintillation tank. The water also provides shielding against ambient radioactivity.

Neutrinos above the threshold energy of $1.8 \mathrm{MeV}$ will be detected by the reaction $\bar{\nu}_{e}+\mathrm{H} \rightarrow e^{+}+n$. The observable energy from this reaction will equal the positron kinetic energy augmented by the $1.022 \mathrm{MeV}$ resulting from essentially calorimetric detection of the positron annihilation gamma rays. Following thermalization of the emitted neutron, an additional $8 \mathrm{MeV}$ will 


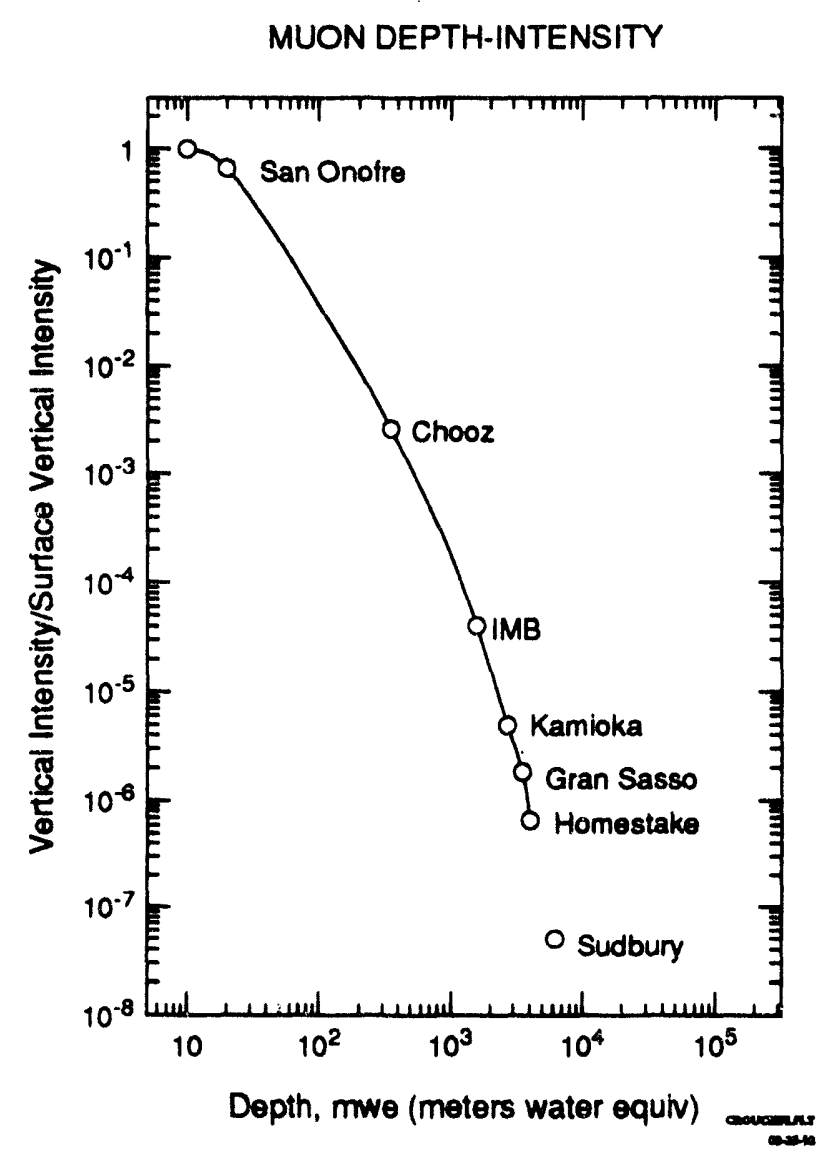

Figure 14: Attenuation of muon background vs depth underground.

be detected as a result of capture of the thermalized neutron by a gadolinium nucleus (total time required $\sim 50 \mu \mathrm{s}$ ). Thus, a readily recognizable delayed coincidence pulse pair will signal the neutrino interaction. The $8 \mathrm{MeV}$ neutron capture event will be well separated from the beta and gamma radiation accompanying decay of members of the ubiquitous uranium and thorium decay chains and from the beta decay of ${ }^{40} \mathrm{~K}$. 
Table 2: Inverse beta decay event rate in the Chooz experiment.

\begin{tabular}{|l|l|}
\hline Reactor power (2 units) & $8.40 \mathrm{GW}$ - thermal \\
Energy release per fission & $203 \mathrm{MeV}$ \\
$\bar{\nu}_{e}$-H cross section & $6.3 \times 10^{-19} \mathrm{~b} /$ fission \\
Fission rate & $2.6 \times 10^{20}$ fissions $/ \mathrm{s}$ \\
Reactor distance & $1.0 \mathrm{~km}$ \\
Target H density & $7 \times 10^{22}$ atoms $/ \mathrm{cm}^{3}$ \\
Fiducial volume & $6.4 \mathrm{~m}^{3}$ \\
Neutron capture efficiency & $60 \%$ at $4 \mathrm{MeV}$ threshold \\
Gd capture efficiency & $85 \%$ \\
Coincidence efficiency & $92 \%$ \\
Event rate & 23 events/d \\
\hline
\end{tabular}

\subsection{Event Rate and Anticipated Result}

We calculate the expected antineutrino event rate in the Chooz experiment to be about $19 \mathrm{~d}^{-1}$ using the assumptions shown in Table 2. For a 200day run we thus anticipate about 4400 events, allowing statistical errors in the viciniti $\because$ of $2 \%$, assuming a good background measurement can be made before the first reactor starts operation in late 1995. 


\section{The Perry Experiment}

\subsection{Overview}

The Perry experiment will be proposed as a highly sensitive neutrino oscillation search taking advantage of the former IMB Fairport (Ohio) deep underground facility together with the Perry Nuclear Power Reactor, optimally situated at a distance of $12.9 \mathrm{~km}$ from the underground laboratory.

The limits on neutrino vacuum oscillations expected from this experiment are shown in Fig. 13 by the curve labelled 'Perry'. For maximal mixing, the experiment will detect oscillations for values of $\Delta m^{2}>8 \times 10^{-5} \mathrm{eV}^{2}$, covering the two-order-of-magnitude gap between the currently available limits and those obtainable from solar neutrino experiments.

The Perry neutrino detector, as shown in Fig. 15, will consist of a 16-mdiameter cylindrical stainless steel tank shielded by an active veto Čerenkov detector. The tank will be filled with 3 metric kilotons (1 kiloton fiducial) of a highly transparent gadolinium-loaded liquid scintillator. ${ }^{4}$ Scintillation photons from particle interactions in the vessel will be collected by 2400 eight-inch photomultipliers and processed by fast multi-hit adc/tdc's. The detector will have good energy resolution, with about 100 photoelectrons detected per $\mathrm{MeV}$ of ionization energy deposited.

Primary shielding against background is provided by the 550 -meter thick rock overburden, which reduces the surface cosmic ray muon flux by a factor of $2 \times 10^{4}$ as shown in Fig. 14. The residual cosmic ray background is further suppressed by active shielding consisting of a two meter-thick water Čerenkov veto detector completely surrounding the steel scintillation tank. The water also provides effective shielding against ambient radioactivity.

Neutrinos above the threshold energy of $1.8 \mathrm{MeV}$ will be detected by the reaction $\bar{\nu}_{e}+\mathrm{H} \rightarrow e^{+}+n$. The observable energy from this reaction will equal the positron kinetic energy augmented by the $1.022 \mathrm{MeV}$ resulting from essentially calorimetric detection of the positron annihilation gamma rays. Following thermalization of the emitted neutron, an additional $8 \mathrm{MeV}$ will be detected as a result of capture of the thermalized neutron by a gadolinium nucleus (total time required $\sim 50 \mu s$ ). Thus, a readily recognizable delayed coincidence pulse pair will signal the neutrino interaction. The $8 \mathrm{MeV}$ neutron capture event will be well separated from the beta and gamma radiation accompanying decay of members of the ubiquitous uranium and thorium de- 


\section{The Perry Experiment}

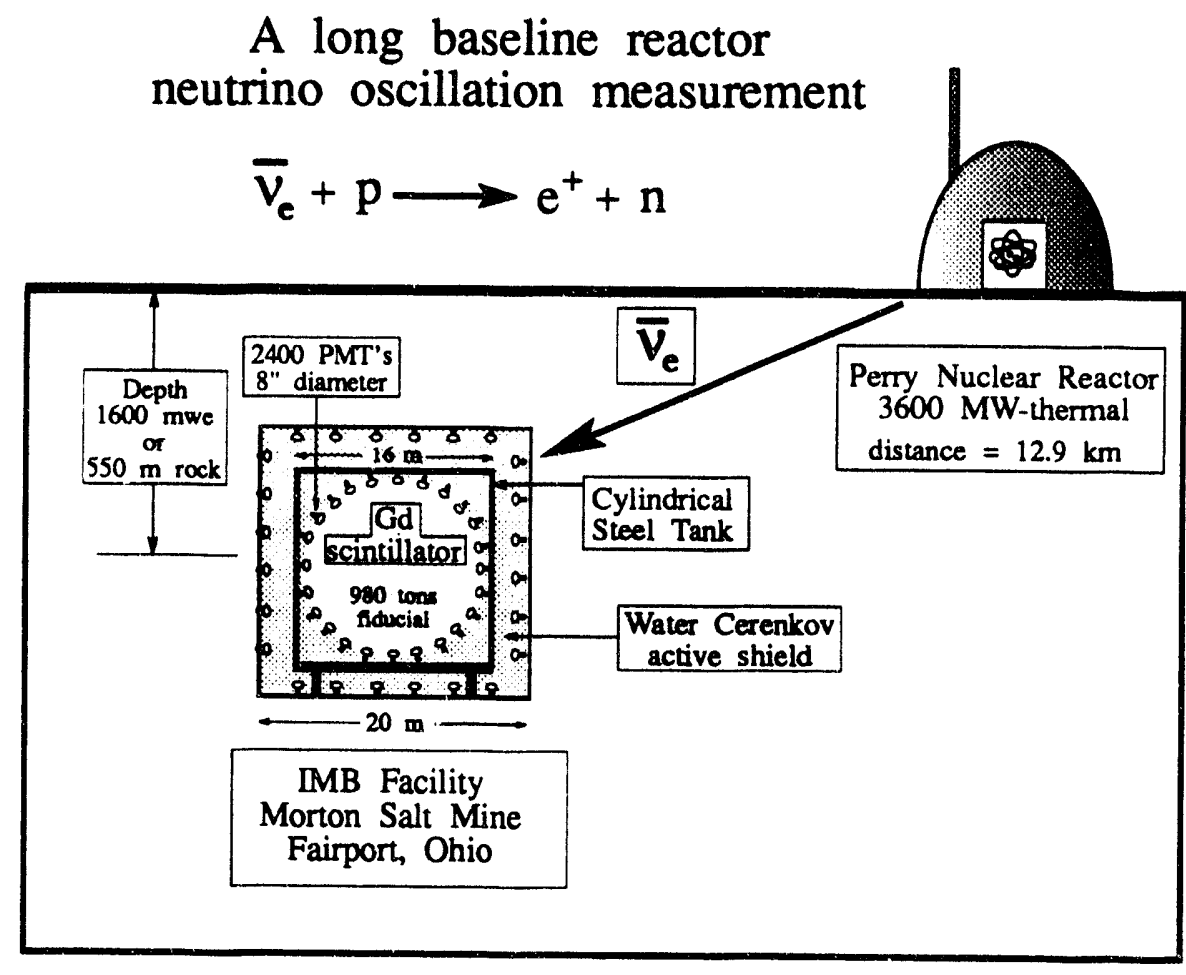

Figure 15: Concept of the Perry neutrino oscillation experiment.

cay chains and from the beta decay of ${ }^{40} \mathrm{~K}$. Further significant rejection of the accidental coincidence background will be possible by reconstructing the positron and neutron capture vertices and requiring a spatial separation not to exceed 2 meters. 


\subsection{Event Rate}

We calculate the expected antineutrino event rate in the Perry experiment to be about $12 \mathrm{~d}^{-1}$ using the assumptions shown in Table 3. For a 1000-day run we thus anticipate more than 10000 events, allowing statistical errors approaching $1 \%$.

Table 3: Inverse beta decay event rate in the Perry experimerit.

\begin{tabular}{|l|l|}
\hline Reactor power & $3.50 \mathrm{GW}-$ thermal \\
Energy release per fission & $203 \mathrm{MeV}$ \\
$\bar{\nu}_{\mathrm{e}}-\mathrm{H}$ cross section & $6.3 \times 10^{-19} \mathrm{~b} /$ fission \\
Fission rate & $1.1 \times 10^{20}$ fissions $/ \mathrm{s}$ \\
Reactor distance & $12.9 \mathrm{~km}$ \\
Target H density & $7 \times 10^{22}$ atoms $/ \mathrm{cm}^{3}$ \\
Fiducial volume & $1150 \mathrm{~m}^{3}$ \\
Gd capture efficiency & $77 \%$ \\
Coincidence efficiency & $90 \%$ \\
Other efficiency factors & $75 \%$ \\
Event rate & 12 events $/ \mathrm{d}$ \\
\hline
\end{tabular}

\subsection{Backgrounds}

Because of the $12.9 \mathrm{~km}$ source-to-detector distance, a major task in the Perry experiment is to reduce the background to the low levels necessary for clean observation of the signal. Fortunately, the delayed coincidence signature for the antineutrino inverse beta decay events, together with a tight spatial coincidence requirement, should obviate the need for the heroic effort required in such singles counting experiments as SNO.

The backgrounds are of three principal types:

Entering Radioactivity Low energy neutrons and gamma rays originating in the rock walls of the facility should be reduced to very low levels by the 
three meters of water shielding. Fission neutrons are attenuated by a factor of $10^{4} \mathrm{iy}$ each meter of water; $2-\mathrm{MeV}$ gamma rays are reduced 170 -fold per meter of water, while at $10 \mathrm{MeV}$ the reduction factor is about 10 per meter. Entering radioactivity should therefore be no problem.

Cosmic Ray Backgrounds Backgrounds induced by through-going cosmic ray muons should be very low because, at the $1570 \mathrm{MWE}$-depth of the Fairport site, the measured muon rate is only $2 \mathrm{~Hz}$ passing through the water Cerenkov detector and therefore about $1 \mathrm{~Hz}$ through the scintillation vessel. By localizing the muon track using information from the Cerenkov detector, and declaring (in software) a veto region around each track, these muons and the delayed beta radioactivity they induce should be strongly suppressed without introduction of significant deadtime.

Stopping muons must also be considered, with $\mu^{-}$probably the most troublesome because of the likelihood of nuclear capture with neutron emission. We estimate the stopping $\mu^{-}$rate in 1000 tons of scintillator to be about $600 \mathrm{~d}^{-1}$. Of these, about 540 will undergo beta decay with a mean life of about $2 \mu \mathrm{s}$ and most of the remaining 60 will be captured with the release of a single neutron. An entering muon veto signal should tag more than $99 \%$ of these events, while time and pulse height cuts should give another factor of at least 10 for the muon beta decays. Again, the background situation looks good.

Another possibly troublesome external background is the interaction of high energy muons in the rock creating a single high energy neutron directed toward the scintillator. The neutron energy required to penetrate the water shield is several hundred $\mathrm{MeV}$. If such a neutron enters the scintillator, the huge recoil proton pulse should allow its rejection. The worst (but unlikely) scenario is for the high energy neutron to lose most of its energy in the water (where the recoil protons would be below Cerenkov threshold) and then to enter the scintillator with an energy of a few MeV. Using the Monte Carlo code GEANT, we are studying this type of event to judge the rate of false correlated events which might be produced.

Internal Radioactivity We expect minimal difficulties with backgrounds to arise from the inevitable internal radioactive contamination of the detector materials; nevertheless, laboratory measurements are in progress aimed at 
evaluating and controlling this source of background.

We have estimated the chance coincidence rate due to internal radioactivity in the Perry detector with the assumption of uranium and thorium levels in the scintillator of $1 \times 10^{-12} \mathrm{~g} / \mathrm{g}$ and in the water shield four times higher. In the positron channel, the singles rate above a threshold of $2 \mathrm{MeV}$ would be $3 \mathrm{~Hz}$, while in the neutron capture channel the rate above $6 \mathrm{MeV}$ would be below $0.01 \mathrm{~Hz}$. In a 100- $\mu$ s gate, therefore, the chance coincidence rate would be $r_{1} r_{2} \tau=3 \mathrm{~d}^{-1}$. With the requirement of a $<2 \mathrm{~m}$ spatial coincidence between the two time-correlated events, the accidental rate would be suppressed by an additional factor of $\sim 30$ to a negligible level.

\subsection{Event Generation and Reconstruction}

To understand and optimize the potential performance of the Perry detector, we are modelling events in the detector using a method combining Monte Carlo techniques (GEANT-based) with ray tracing. The input parameters for the program are listed in Table 4.

The Monte Carlo included the effects of light attenuation, scattering, reflections from PMT's, and PMT timing jitter. Realistic trigger conditions were used in deciding whether an event was to be kept.

Table 4: Input parameters for event reconstruction program.

\begin{tabular}{|l|l|}
\hline Geometry & $16 \mathrm{~m} \times 16 \mathrm{~m}$ cylinder \\
Scintillation efficiency & $150 \mathrm{eV} /$ photon $=45 \%$ of anthracene \\
Scintillator attenuation length & $12 \mathrm{~m}$ \\
PMT type & Hamamatsu R1408 $8^{\prime \prime}$ \\
PMT number & 2400 \\
Light cone factor & 1.8 \\
PMT quantum efficiency & $20 \%$ \\
PMT single pe time spread & $8 \mathrm{~ns}$ FWHM \\
Dynode collection factor & $50 \%$ \\
\hline
\end{tabular}

Event distributions resulting from the reconstruction of one year's worth of inverse beta events in the detector are shown in Fig. 16. The mean number 


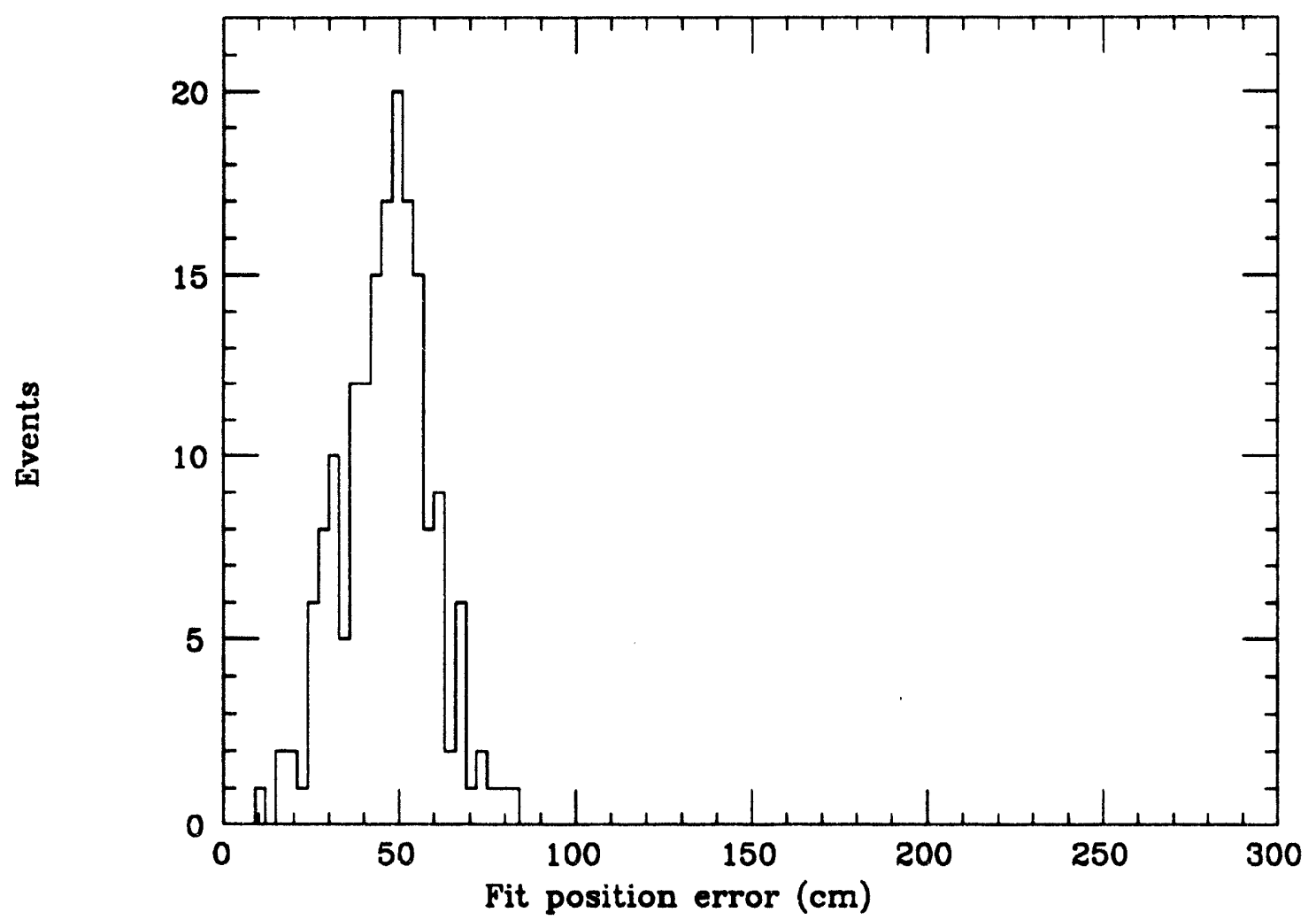

Figure 16: Distance between reconstructed and actual positions in the Monte Carlo for the positron signals in inverse beta decay.

of photoelectrons produced ('PMT hits') is about 90 pe's/MeV. The reconstructed energy distribution has a standard deviation of $4 \%$ for the neutron capture events, while the mean reconstruction position error is about $50 \mathrm{~cm}$. These figures are excellent, indicating that this detector configuration would be adequate for the experiment. 


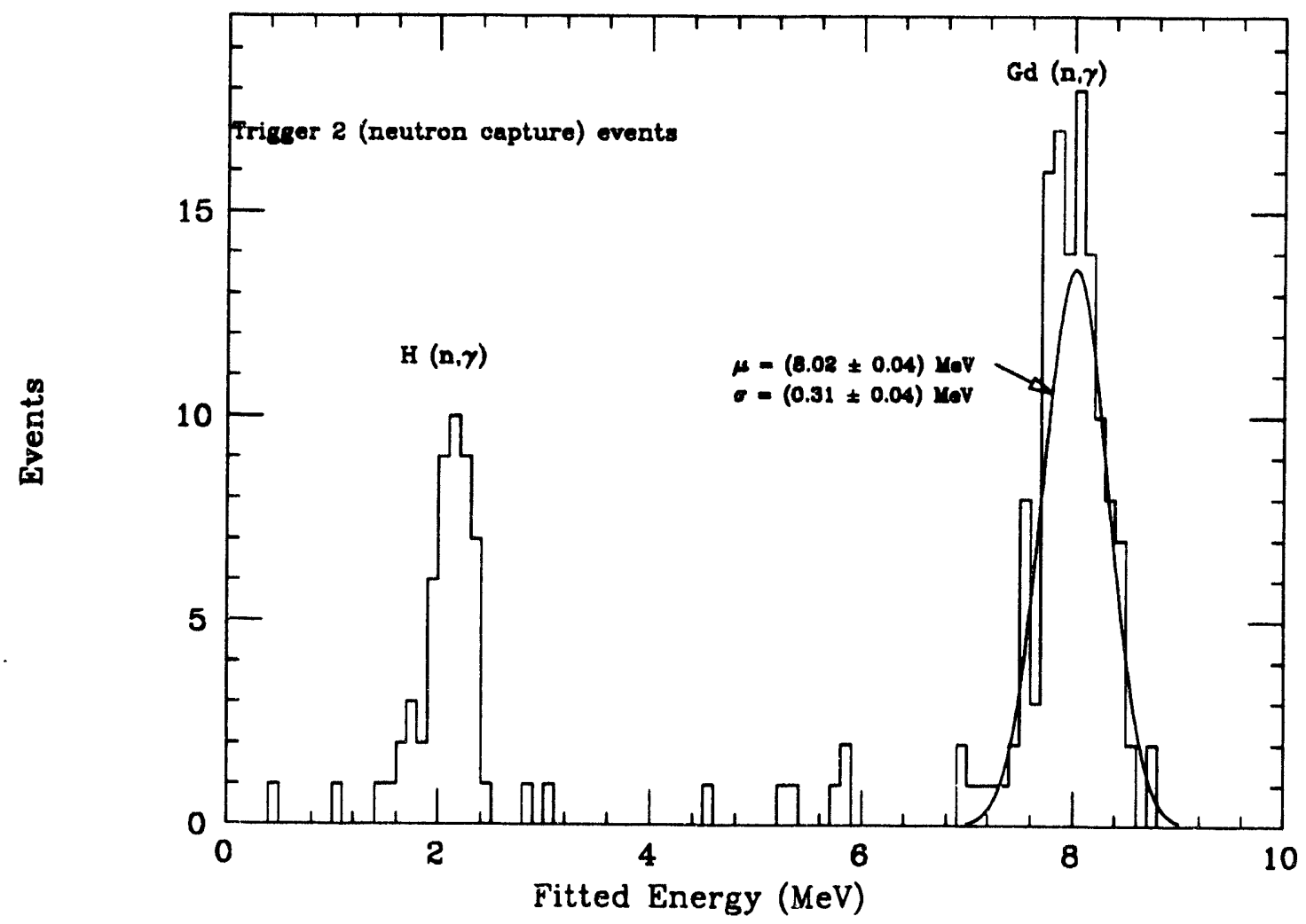

Figure 17: Reconstructed neutron capture energy after inverse beta decay. Most of the captures are on gadolinium $(8 \mathrm{MeV})$; however capture on hydrogen $(2.2 \mathrm{MeV})$ is also clearly seen. 


\subsection{Background Tests}

Although laboratory studies can help to determine the design parameters in an experiment such as this one, only on-site measurements can truly gauge the extent of background problems. For this reason, the Chooz experiment (section 4) will provide an excellent testing ground for our design. In addition, we plan to refurbish, set up and run an existing seven ton gadoliniumloaded liquid scintillator tank to monitor neutrons and gamma rays at the Morton mine experimental site and to measure the ambient radon and perhaps thoron levels. In addition, we plan to use a five-inch $\mathrm{NaI}(\mathrm{Tl})$ detector for gamma surveys of the site.

With data from these measurements, together with experience gained from the Chooz experiment, we will be in a very good position to proceed with plans for the Perry detector without risk of unpleasant surprises. We plan to submit the completed proposal for the Perry experiment by the middle of 1993. 


\section{SSC-Related Research}

\subsection{Introduction}

Drexel is a participating institution in the GEM collaboration for the SSC. The GEM detector focuses on the detection of gammas, electrons and muons, with an emphasis on calorimetry. The past year has been one of testing calorimeter options, and narrowing the field. This process is not quite complete, but the final decisions are expected soon.

\subsubsection{SSC R\&D}

There has been some $\mathrm{R} \& \mathrm{D}$ approved in advance of the GEM detector approval. One of us (C.L.) is part of the SSCintCal group for scintillating fiber calorimetry for the SSC, which received SSC R\&D and Texas National Research Laboratory Commission funding.

The scope of the R\&D effort at Drexel is currently quite limited; a liquid scintillator is being developed for use in liquid-fiber forward calorimeters. The forward calorimeters are made of grooved tungsten plates with $\mathrm{MgF}_{2}$ coatings. Light from the scintillator is brought out by total internal reflection. The evaluation criteria for the liquid scintillators that are of interest for liquid-fiber calorimetry are a high refractive index (to improve light collection) and tolerance of a large radiation dose.

We currently have a refractometer on long-term loan from the Drexel Chemistry department, and have tested some candidate scintillators. There is a considerable amount of overlap between the scintillators of interest for liquid-fiber calorimeters and for our other liquid scintillator detector projects.

Irradiation tests of liquid scintillator samples were performed this past year, and no degradation of the scintillators was observed.

The liquid-fiber calorimeter itself is being developed at Texas A\&M, and beam tests are expected in the next year. The technology of liquid-fiber calorimeters is applicable to all SSC detector forward calorimeters where the issue of radiation damage is foremost.

\subsubsection{GEM Electronics}

While the detector development is still at an early stage, we expect that our future involvement will be in the area of electronics for GEM. 
Our experience with electronics for MACRO should allow us to make a useful contribution to the trigger electronics on the GEM experiment. In particular, the development of fast energy and $p_{T}$ triggers is an area that we find of particular interest.

A fast ADC/TDC multi-hit system based on flash-ADCs is in the early development stages this past year. This system shows promise for application in GEM electronics if its overall size can be decreased to the 1-2 chip level per channel.

We have been investigating the use of thick-film hybrid facilities available on campus for this purpose, but the full-custom chip approach looks more promising for eventual use on the SSC. We have recently enhanced our ability to design custom electronics by the acquisition of the MAGIC program for chip-design and layout. 


\section{Recent Drexel Particle Physics Publica- tions}

1. First Results from the MACRO Experiment at the Gran Sasso Laboratory, The MACRO Collaboration, Nucl. Phys. B19 (1991) 128.

2. Cosmic Ray Search for Strange Quark Matter with the MACRO Detector, The MACRO Collaboration, Nucl. Phys. B24 (1991) 191.

3. Study of the Primary Cosmic Ray Composition with the MACRO Experiment at Gran Sasso, The MACRO Collaboration, in Proc. 9o.nd Int'l. Cosmic Ray Conf., (Dublin) 2 (1991) 1.

4. Study of High Energy Cosmic Rays through the Measurement of the Electromagnetic and TeV Muon Components of Extensive Air Showers by EAS-TOP and MACRO, The EAS-TOP and MACRO Collaborations, in Proc. 22nd Int'l. Cosmic Ray Conf., (Dublin) 2 (1991) 61.

5. A Search for Prompt Muons using the MACRO Detector at Gran Sasso, The MACRO Collaboration, in Proc. 22nd Int'l. Cosmic Ray Conf., (Dublin) 4 (1991) 169.

6. Measurement of Electromagnetic and TeV Muon Components of Extensive Air Showers by EAS-TOP and MACRO, The MACRO and EASTOP Collaborations, in Proc. 22nd Int'l. Cosmic Ray Conf., (Dublin) 4 (1991) 563.

7. Measurement of the Muon Decoherence Function with the MACRO Experiment at Gran Sasso, The MACRO Collaboration, in Proc. 2ind Int'l. Cosmic Ray Conf., (Dublin) 4 (1991) 567.

8. Arrival Time Distributions of Very High Energy Cosmic Ray Muons in MACRO, The MACRO Collaboration, in Proc. 22nd Int'l. Cosmic Ray Conf., (Dublin) 4 (1991) 571.

9. Search for Astrophysical Point Sources of Muons with the MACRO Detector, The MACRO Collaboration, in Proc. 22nd Int'l. Cosmic Ray Conf., (Dublin) 4 (1991) 591. 
10. A Search for Sidereal Anisotropies with Underground Muons and an All-Sky Survey for Muon Point Sources with the MACRO Detector, The MACRO Collaboration, in Proc. 22nd Int'l. Cosmic Ray Conf., (Dublin) 4 (1991) 595.

11. The GRANDE Experiment, The GRANDE Collaboration, in Proc. 22nd Int'l. Cosmic Ray Conf., (Dublin) 4 (1991) 690.

12. Stellar Gravitational Collapse Detection by MACRO: Characteristics and Results, The MACRO Collaboration, in Proc. 22nd Int'l. Cosmic Ray Conf., (Dublin) 4 (1991) 698.

13. Search for Nuclearites with the MACRO Detector, The MACRO Collaboration, in Proc. 22nd Int'l. Cosmic Ray Conf., (Dublin) 4 (1991) 730 .

14. Coincidence of EM and TeV Muon Components of EAS with the EASTOP and MACRO Detectors, The MACRO and EAS-TOP Collaborations, in The Vancouver Meeting, Particles 66 Fields '91, eds. D. Axen, D. Bryman and M. Comyn (World Scientific, Singapore, 1992), p. 903.

15. MACRO Multi-Muon Event Analysis, The MACRO Collaboration, in The Vancouver Meeting, Particles \& Fields '91, eds. D. Axen, D. Bryman and M. Comyn (World Scientific, Singapore, 1992), p. 906.

16. Muon Astronomy Results from MACRO, The MACRO Collaboration, in The Vancouver Meeting, Particles \& Fields '91, eds. D. Axen, D. Bryman and M. Comyn (World Scientific, Singapore, 1992), p. 1014.

17. Senrch for Nuclearites with the MACRO Detector, The MACRO Collaboration, in The Vancouver Meeting, Particles \& Fields '91, eds. D. Axen, D. Bryman and M. Comyn (World Scientific, Singapore, 1992), p. 1017.

18. Study of Neutral Currents with MACRO, The MACRO Collaboration, in Proc. 2nd Int'l. Conf. on Trends in Astroparticle Physics, (Aächen, Germany, Oct. 1991). 
19. Results from MACRO, The MACRO Collaboration, in Proc. 2nd Int'l. Conf. on Trends in Astroparticle Physics, (Aächen, Germany, Oct. 1991).

20. A New Detector: Borexino, The Borex Collaboration, in Proc. 3rd Int'l. Workshop on Neutrino Telescopes, (Venice, Feb. 1991), p. 73.

21. Improvements in the CR39 Polymer for the MACRO Experiment at the Gran Sasso Laboratory, The MACRO Collaboration, Nucl. Tracks Radiat. Meas. 19 (1991) 641.

22. Aspects of Experimental Particle Physics from Below: Recent Results and Future Prospects for the MACRO Experiment, The MACRO Collaboration, in Proc. 15th Johns Hopkins Workshop on Current Problems in Particle Theory, (Baltimore, August 1991).

23. The Borexino Project to Study Solar Neutrinos, The Borexino Collaboration, in Massive Neutrinos and Tests of Fundamental Symmetries, eds. O. Fackler, G. Fontaine and J. Tran Thanh Van (Editions Frontières, Gif-sur-Yvette, France, 1992), p. 37.

24. The Borexino Solar Neutrino Experiment, The Borexino Collaboration, in Proc. XXVIIth Rencontre de Moriond, Les Arcs, France, Jan. 1992, eds. O. Fackler, G. Fontaine and J. Tran Thanh Van (Editions Frontières, Gif-sur-Yvette, France).

25. Search for WIMP Signals from the Center of the Earth, The MACRO Collaboration, in Proc. 2nd Int'l. Conf. on Gamma Ray and Neutrino Cosmology, ed. D. Cline (World Scientific, Singapore).

26. Stellar Collapse Detection with MACRO, The MACRO Collaboration, in Proc. 2nd Int'l. Conf. on Gamma Ray and Neutrino Cosmology, ed. D. Cline (World Scientific, Singapore).

27. Perry: A Long Baseline Reactor Neutrino Oscillation Experiment, R.I. Steinberg, C.E. Lane, M. Mittelbrunn and F.C. Wang, in Proc. 2nd Int'l. Conf. on Gamma Ray and Neutrino Cosmology, ed. D. Cline (World Scientific, Singapore). 
28. Arrival Time Distributions of Very High Energy Cosmic Ray Muons in MACRO, The MACRO Collaboration, Nucl. Phys. B370 (1992) 432.

29. Study of the Ultra-High Energy Primary Cosmic Ray Composition with the MACRO Experiment, The MACRO Collaboration, Phys. Rev. D46 (1992) 895.

30. Search for Neutrino Bursts from Collapsing Stars with the MACRO Detector, The MACRO Collaboration, Astropart. Phys., 1992, accepted.

31. The MACRO Detector at Gran Sasso: The First Supermodule, The MACRO Collaboration, Nucl. Instrum. Meth., 1992, accepted.

32. Stellar Gravitational Collapse Detection by MACRO: Characteristics and Results, The MACRO Collaboration, Nucl. Phys. B, 1992, accepted.

33. Search for Strange Quark Matter Using the MACRO Detector, The MACRO Collaboration, Phys. Rev. Lett., 1992, submitted.

34. Measurement of the Decoherence Function with the MACRO Detector at Gran Sasso, The MACRO Collaboration, Phys. Rev. D, 1992, accepted.

35. Search for Strange Quark Matter Using the MACRO Detector, The MACRO Collaboration, Phys. Rev. Lett., 1992, submitted.

36. Muon Astronomy with the MACRO Detector, The MACRO Collaboration, Ap. J., 1992, to be submitted.

37. Proceedings of the 1992 Franklin Symposium: In Celebration of the Discovery of the Neutrino, eds. C.E. Lane and R.I. Steinberg (World Scientific, 1992), to be published.

38. The Perry Experiment: A Long Baseline Reactor Neutrino Oscillation Search, R.I. Steinberg, C.E. Lane, M. Mittelbrunn and F.C. Wang, in Proceedings of the 1992 Franklin Symposium: In Celebration of the Discovery of the Neutrino, eds. C.E. Lane and R.I. Steinberg (World Scientific, 1992). 
39. PERRY: A Reactor Neutrino Oscillation Experiment Sensitive to $\Delta m^{2}$ $=10^{-4} \mathrm{eV}^{2}$, R.I. Steinberg, C. Lane, M. Mittelbrunn and F.C. Wang, in Particle Astrophysics, eds. O. Fackler, G. Fontaine and J. Tran Thanh Van (Editions Frontières, Gif-sur-Yvette, France).

40. A MACRO Sampler, The MACRO Collaboration, in Proc. Int'l. Conf. on High Energy Physics, Dallas, August 1992.

41. A Neutrino Oscillation Experiment Using the Perry Reactor and a Large Neutron Capture Scintillation Detector, invited talk by R.I. Steinberg, Workshop on Future Experiments at the Fairport Facility, Boston, June 1991.

42. The Perry Experiment, invited talk by R.I. Steinberg, IMB Collaboration Meeting, Irvine, CA, Jan. 1992.

43. The Perry Experiment, a Long Baseline Reactor Neutrino Oscillation Search, invited talk by R.I. Steinberg, 2nd Int'l. Conf. on Gamma Ray and Neutrino Cosmology, Los Angeles, Feb. 1992.

44. The Perry Experiment, invited talk by R.I. Steinberg, Franklin Symposium in Celebration of the Discovery of the Neutrino, Philadelphia, May 1992.

45. Improved Neutron Detection for MACRO, invited talk by R.I. Steinberg, Workshop on Possible Future Enhancements of the MACRO Experiment, Yarmouthport, MA, May 1992.

46. Results from and Prospects for MACRO, invited talk by R.I. Steinberg, IVth Rencontre de Blois, Particle Astrophysics, Chateau de Blois, France, June 1992.

47. The Perry Experiment: A Long Baseline Reactor Neutrino Oscillation Search, invited talk by R.I. Steinberg, IVth Rencontre de Blois, Particle Astrophysics, Chateau de Blois, France, June 1992.

48. A Long Baseline Reactor Neutrino Oscillation Search, invited talk by R.I. Steinberg, Workshop on Future Experiments at the IMB Fairport Underground Laboratory, University of New Mexico, Albuquerque, NM, Sept. 1992. 
49. Perry: A New Probe for Neutrino Oscillations, invited talk by R.I. Steinberg, First Symposium on Nuclear Physics in the Universe, Oak Ridge, TN, Sept. 1992.

50. Muon Astronomy with the MACRO Detector, The MACRO Collaboration, Bull. Am. Phys. Soc. 36, 1382 (1991).

51. Experimental Particle Astrophysics: A Report on the MACRO experiment, The MACRO Collaboration, Bull. Am. Phys. Soc. 36, 1697 (1991).

52. Recent Results and Future Prospects for the MACRO Experiment, The MACRO Collaboration, Int'l. School for Astroparticle Physics, Woodlands, TX, Jan. 1991.

53. Multiple Muon Physics with MACRO, The MACRO Collaboration, Lepton-Photon and High Energy Physics Conference, Geneva, July 1991.

54. Muon Astronomy in MACRO, The MACRO Collaboration, LeptonPhoton and High Energy Physics Conference, Geneva, July 1991.

55. Search for Strange Quark Matter in MACRO, The MACRO Collaboration, Lepton-Photon and High Energy Physics Conference, Geneva, July 1991.

56. Preliminary Radon Measurements in the Gran Sasso Underground Laboratory, B.B. Bam, A. Bassignani, P. Finazzi, G. Giacomelli, L. Lembo, G. Maltoni, L. Patrizii, F. Predieri, C. Sartorio, P. Serra, R. Steinberg and V. Togo, 2nd Workshop on Radon Monitoring, Trieste, Nov. 1991.

57. Muon Astronomy with MACRO, The MACRO Collaboration, Workshop on High Energy Neutrino Astrophysics, Honolulu, March 1992.

58. Neutrino Astronomy with MACRO, The MACRO Collaboration, Workshop on High Energy Neutrino Astrophysics, Honolulu, March 1992.

59. Transparencies from the Franklin Symposium in Celebration of the Discovery of the Neutrino, Philadelphia, April 1992, eds. C.E. Lane and R.I. Steinberg (Drexel University, May 1992). 
60. Siellar Gravitational Collapse Detection by MACRO: Characteristics and Results, The MACRO Collaboration, Neutrino '92, Grenada, June 1992.

61. Status and Prospects of the MACRO Experiment, The MACRO Collaboration, Summer Institute on Low Energy Neutrinos in Physics and Astrophysics, l'Aquila, Italy, July 1992.

62. Multiple Muons with MACRO, The MACRO Collaboration, European Conf. on Cosmic Ray Physics, Geneva, July 1992.

63. Effect of the Geomagnetic Field on the Separation of Underground Muons, The MACRO Collaboration, European Conf. on Cosmic Ray Physics, Geneva, July 1992.

64. Muon Astronomy with MACRO, The MACRO Collaboration, European Conf. on Cosmic Ray Physics, Geneva, July 1992.

65. Muon and Neutrino Astronomy (MACRO), The MACRO Collaboration, XXVIth Int'l. Conf. on High Energy Physics, Dallas, August 1992.

66. Monopoles and Strange Quark Matter Search (MACRO), The MACRO Collaboraiion, XXVIth Int'l. Conf. on High Energy Physics, Dallas, August 1992.

67. Borexino: A Real Time Detector for Low Energy Solar Neutrinos, The Borexino Collaboration, XXVIth Int'l. Conf. 'sn High Energy Physics, Dallas. August 1992. 


\section{References}

[1] J. Petrakis, Muon Astronomy with a Large Underground Detector, Ph.D. thesis, Indiana University, (1990).

[2] The MACRO Collaboration, Search for Neutrino Bursts from Collapsing Stars with the MACRO Detector, Astroparticle Physics, (1992) accepted.

[3] The MACRO Collaboration, Arrival Time Distributions of Very High Energy Cosmic Ray Muons in MACRO, Nucl. Phys. B370 (1992) 432.

[4] The MACRO Collaboration, Study of the Ultra-High Energy Primary Cosmic Ray Composition with the MACRO Experiment, Phys. Rev. D (1992) accepted.

[5] The MACRO Collaboration, Cosmic Ray Search for Strange Quark Matter with the MACRO Detector, Nucl. Phys. B24 (1991.) 191.

[6] The MACRO Collaboration, The MACRO Detector at Gran Sasso: First Supermodule, Nucl. Instrum. Meth. (1992) accepted.

[7] J.G. Learned, S. Pakvasa and T.J. Weiler, Neutrino Mass and Mixing Implied by the Underground Deficit of Low Energy Muon-Neutrino Events, Phys. Lett. B207 (1988) 79.

[8] The MACRO Collaboration, The MACRO Detector at the Gran Sasso Laboratory, Nucl. Instrum. Meth. A264 (1988) 18.

[9] M.S. Turner, E.N. Parker and T.J. Bogdan, Magnetic Monopoles and the Survival of Galactic Magnetic Fields, Phys. Rev. D26 (1982) 1296.

[10] S.P. Ahlen, T.M. Liss, C. Lane and G. Liu, Scintillation from Slow Protons: A Probe of Monopole Detection Capabilities, Phys. Rev. Lett. 55 (1985) 181.

[11] D.J. Ficenec, S.P. Ahlen, A.A. Marin, J.A. Musser and G. Tarlé, Observation of Electronic Excitation by Extremely Slow Protons with Applications to the Detection of Supermassive Charged Particles, Phys. Rev. D36 (1987) 311. 
[12] X. Wen and E. Witten, Electric and Magnetic Charges in Superstring Models, Nucl. Phys. B261 (1985) 651.

[13] Z. Maki et al., Prog. Theor. Phys. 284, (1962) 870.

[14] B. Pontecorvo, Sov. Phys. JETP 6, (1958) 429.

[15] G. Zacek et al., Neutrino-Oscillation Experiments at the Gösgen Nuclear Power Reactor, Phys. Rev. D34 (1986) 2621. 

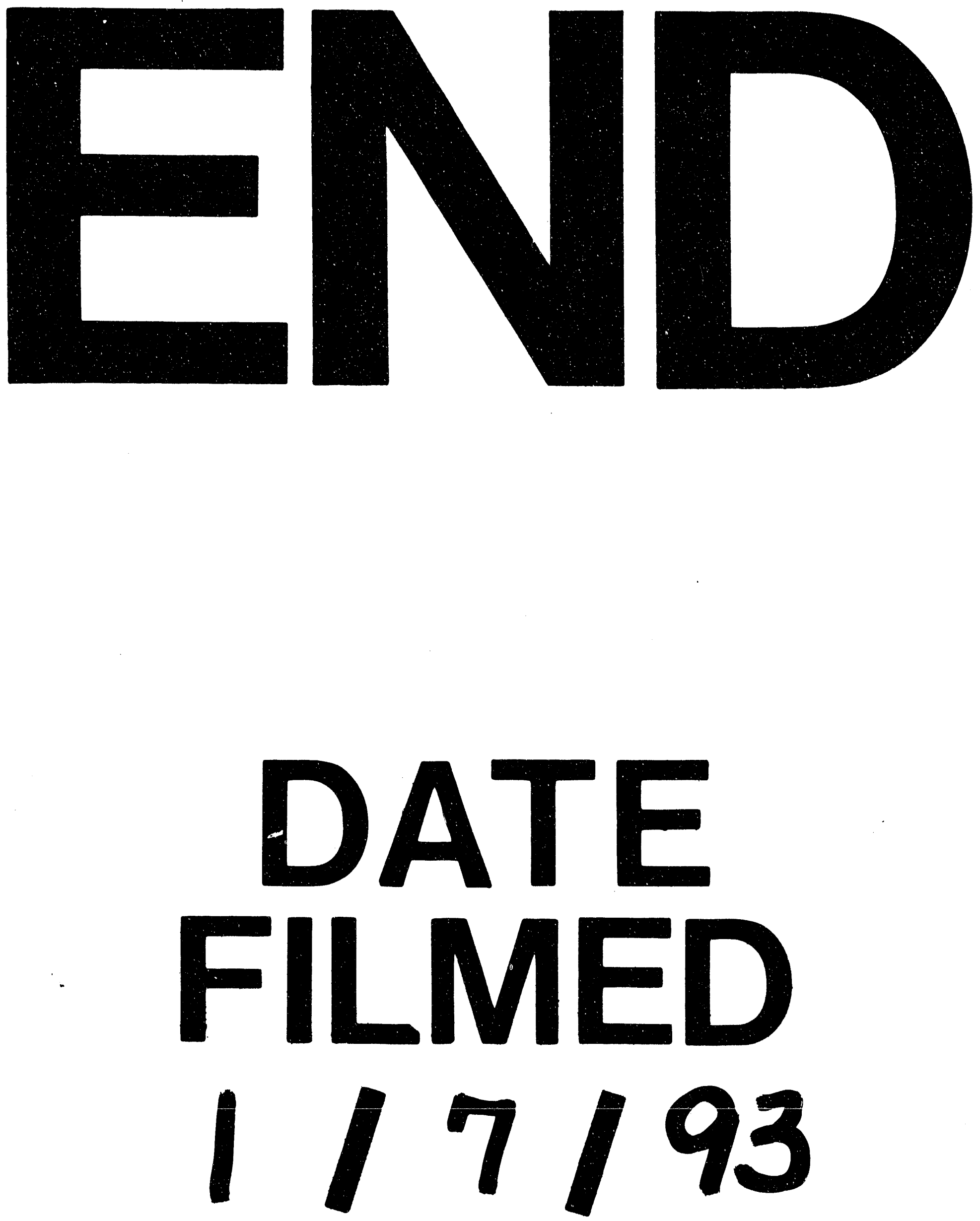
